\title{
APOCINÁCEAS DE AYER Y HOY. CONOCIMIENTO HISTÓRICO Y REEVALUACIÓN DE LA DIVERSIDAD Y DISTRIBUCIÓN DE APOCYNACEAE EN MÉXICO
}

\section{APOCYNACEAE OF YESTERDAY AND TODAY. HISTORICAL KNOWLEDGE AND REEVALUATION OF THE DIVERSITY AND DISTRIBUTION OF APOCYNACEAE IN MEXICO}

\author{
@Leonardo O. Alvarado-Cárdenas*, @lucio lozada-Pérez, @C. Sofia Islas-Hernández, @Ericka B. Cortez, \\ OKaren G. Maya-Mandujano, @María G. Chávez-Hernández
}

\begin{abstract}
Departamento de Biología Comparada, Laboratorio de Plantas Vasculares, Facultad de Ciencias, Universidad Nacional Autónoma de México, México
\end{abstract}

*Autor de correspondencia: leonardoac@ciencias.unam.mx

\begin{abstract}
Resumen
Antecedentes: La familia Apocynaceae está entre las 13 más diversas de México y su conocimiento taxonómico ha tenido una atención creciente con la implementación de filogenias y otras herramientas para resolver su sistemática. Con más de 10 años del último listado enfocado en la familia, resulta necesario actualizar el conocimiento sistemático del grupo.

Pregunta: ¿Cómo se ha consolidado hasta nuestros días el conocimiento sistemático del grupo en México? ¿Cómo ha cambiado el número de géneros y especies de Apocynaceae en el país y su distribución geográfica por estado?

Especie de estudio: Apocynaceae

Sitio de estudio: México

Método: Revisión de herbarios nacionales e internacionales, bases de datos y una búsqueda intensiva de literatura. Así como colectas en diferentes partes del país.

Resultados: Apocynaceae presenta 52 géneros y 418 especies nativas, 204 de ellas endémicas. La subfamilia Asclepiadoideae destaca con 27 géneros y 315 especies. Los estados con más especies y endemismos son Oaxaca, Chiapas, Veracruz y Guerrero. Los estados con más microendemismos son Oaxaca (17 spp.), Chiapas y Jalisco (7 spp.), Baja California Sur y Veracruz (5 spp.). Se proporciona una clave de géneros y un listado actualizado.

Conclusiones: Apocynaceae se ubica en el lugar número 12 de las familias más diversas del país. Esto reitera al país como un centro de diversidad del grupo. La integración de más estudios relacionados a la aplicación de herramientas filogenéticas, modelos de distribución y redes de interacción biológica permitirá entender mejor a la familia y proporcionar una historia natural más completa.
\end{abstract}

Palabras clave: Asclepiadoideae, conservación, endemismo, sistemática.

\begin{abstract}
Background: In Mexico, Apocynaceae is among the 13 most diverse families. The taxonomic knowledge of the family has had increasing attention with the implementation of phylogenies and other tools to solve its systematics. Therefore, more than 10 years after the last list focused on the family, it is necessary to update the systematic knowledge of the group.

Questions: How the systematic knowledge of the group has been consolidated to this day? Compared to what we knew 10 years ago of Apocynaceae in Mexico, has the number of genera and species changed? Is so, how? And what's their geographical distribution by state?

Species study: Apocynaceae

Study site and dates: Mexico

Methods: Revision of national and international herbaria, databases and an intensive literature search. As well as collecting in different parts of the country.

Results: Apocynaceae registered 52 genera and 418 native species, 204 of them are endemic. The Asclepiadoideae subfamily contributed 27 genera and 315 species. The states with more species and endemisms are Oaxaca, Chiapas, Veracruz and Guerrero. The states with more microendemisms are Oaxaca (17 spp.), Chiapas and Jalisco (7 spp.), and Baja California Sur and Veracruz (5 spp.).

Conclusions: Apocynaceae is in the 12th place of the most species diverse families in Mexico. This reiterates the country as a center of apocynaceous diversity. The integration of people interested in the group, the application of phylogenetic tools, distribution models and biological interaction networks will allow us to better understand the family and provide a more complete natural history.
\end{abstract}

Keywords: Asclepiadoideae, conservation, endemism, systematics. 
La familia Apocynaceae se encuentra entre los grupos más diversos del mundo con alrededor de 4,500 especies (Rapini 2012, Endress et al. 2014, 2018). Es un grupo monofilético dentro del orden Gentianales junto con Gelsemiaceae, Gentianaceae, Loganiaceae y Rubiaceae (Stevens 2001, APG IV 2016, Yang et al. 2016). Se distingue del resto de las familias por la presencia de laticíferos, ovario bicarpelar, apocárpico y súpero, presencia de un ginostegio (fusión del androceo y gineceo), polinios en muchas de sus especies, además de frutos foliculares y semillas comosas (Endress \& Bruyns 2000, Juárez-Jaimes et al. 2007). Actualmente se reconocen tres subfamilias, Asclepiadoideae, Periplocoideae y Secamonoideae; y dos grupos informales, no monofiléticos: las rauvolfioides $\mathrm{y}$ apocynoides, que correspondían a Rauvolfioideae y Apocynoideae (Simões et al. 2016) y que aún se reconocen de forma provisional debido a sus atributos morfológicos distintivos (Tabla 1). La familia tiene una distribución pantropical y muchas de sus especies cuentan con importancia económica, medicinal o cultural (Gaillard et al. 2004, Wong et al. 2013). Algunos de los centros de mayor diversidad del grupo se encuentran en Brasil, China, México y Sudáfrica, cada uno con más de 400 especies (Ping-Tao et al. 1995a, $\underline{b}$, Bruyns 2005, BFG 2015, Villaseñor 2016). El conocimiento taxonómico de Apocynaceae ha tenido una creciente atención con la implementación de filogenias con datos moleculares y morfológicos, así como de otras herramientas para resolver la sistemática dentro de la familia, esto ha generado numerosos cambios en la circunscripción de muchos de sus taxones.

En México, las Apocynaceae están representadas por los grupos rauvolfioides, apocynoides y Asclepiadoideae

Tabla 1. Comparación de los atributos generales de los grupos de Apocynaceae presentes en México. Las ilustraciones corresponden a Aspidosperma megalocarpon, Prestonia portobellensis y Asclepias melantha. Acuarelas de Aldi de Oyarzabal.

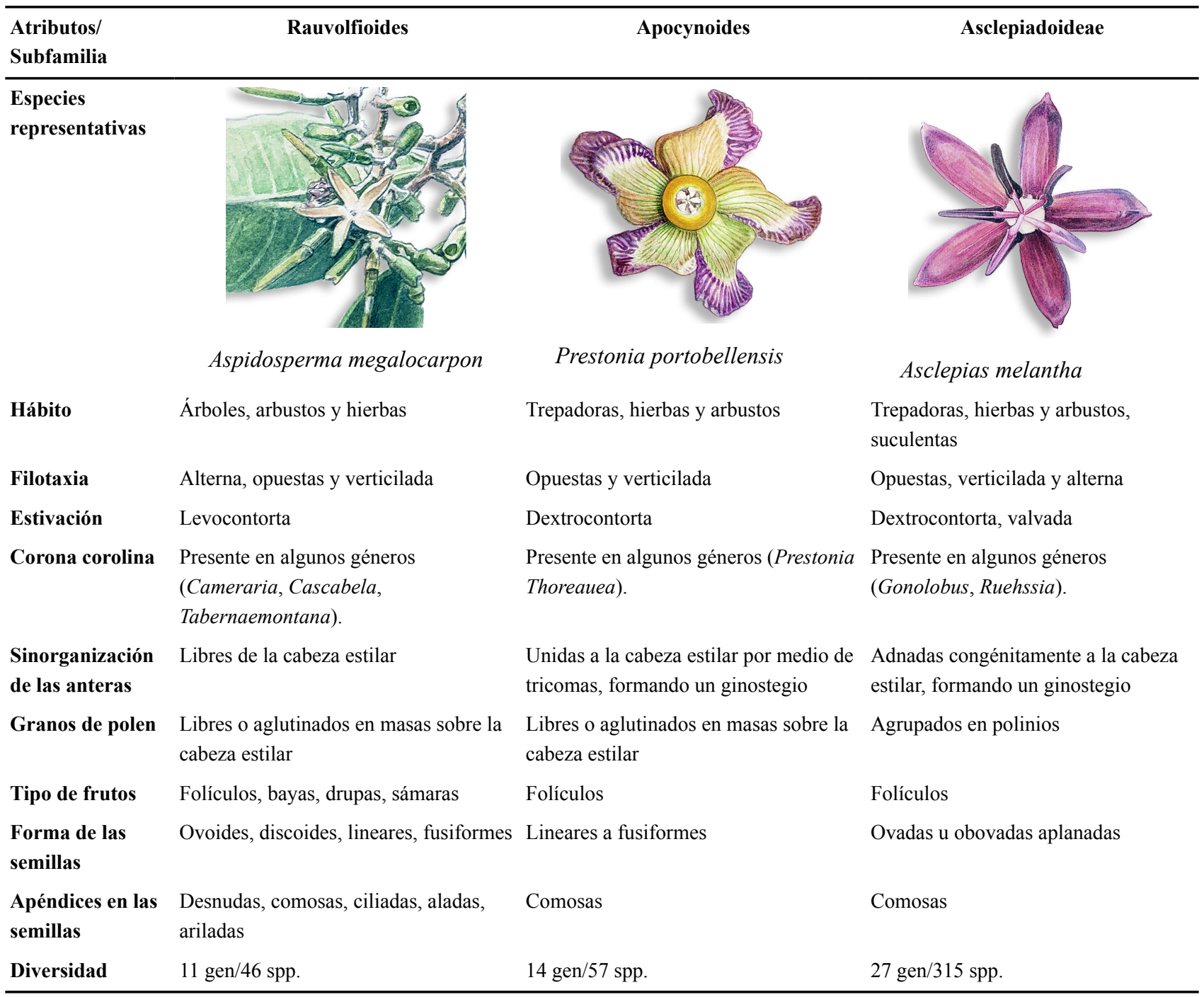


(figuras 1- $\underline{3}$, Tabla 1) (Juárez-Jaimes et al. 2007) y se ubica entre las 13 familias con más diversidad específica (Villaseñor 2016, Sosa et al. 2018). El grupo constituyen un elemento típico en la composición del bosque tropical caducifolio (Rzedowski \& Calderón de Rzedowski 2013) y es la familia con más especies de lianas en México (IbarraManríquez et al. 2015); además, muchas de sus especies tienen importancia económica y cultural (Fernández-Brewer et al. 2008, Alvarado-Cárdenas et al. 2017).

El conocimiento del grupo en México cuenta con importantes aportes taxonómicos, por ejemplo, listados de especies para diferentes estados del país (Williams 1996, Alvarado-Cárdenas 2004, Juárez-Jaimes et al. 2007, Alvarado-Cárdenas \& Juárez-Jaimes 2011), revisiones a nivel genérico (Juárez-Jaimes \& Ángeles-Trujillo 2013, Alvarado-Cárdenas \& Morales 2014, Alvarado-Cárdenas et al. 2017, 2019) y tratamientos taxonómicos regionales (Rodríguez-Morales 2015, Cortez-Castro 2018, HernándezBarón et al. 2019), así como descripciones de nuevas especies (i.e., Williams 2002a, Alvarado-Cárdenas 2004, 2007, Fishbein 2008, Juárez-Jaimes \& Alvarado-Cárdenas 2010, Lozada-Pérez 2010, Alvarado-Cárdenas \& JuárezJaimes 2012, Lozada-Pérez \& Diego-Pérez 2012, JuárezJaimes \& Lozada-Pérez 2015). A la par, se han realizado cambios en la circunscripción taxonómica de varios grupos, apoyados en análisis filogenéticos y morfológicos (Simões et al. 2010, 2016, Morillo 2015, Stevens 2018, do Espírito Santo et al. 2019).

Entre los estudios recientes de la familia en México se encuentran el trabajo de Juárez-Jaimes et al. (2007) quienes reportan 51 géneros y 385 especies nativas, pero a más de 10 años de su publicación, es necesaria una actualización que integre los cambios taxonómicos posteriores (Endress et al. 2014, 2018), así como numerosos taxones descritos. Por su parte, Villaseñor (2016) publicó un listado de la flora vascular de México, en donde registró 58 géneros y 418 especies, pero varios de los taxones incluidos son sinónimos y no se consideran numerosas adiciones, nuevos registros y correcciones de la distribución geográfica de varias de las especies. Además, se carece de una clave genérica actualizada e integral de la diversidad de Apocynaceae para el país, ya que las disponibles incluyen sólo algunas de las subfamilias (Standley 1926, Williams 1996) o corresponden a tratamientos regionales con una representación limitada de los géneros (Juárez-Jaimes \& Lozada-Pérez 2003, Alvarado-Cárdenas 2004, Stevens \& Morales 2009, CortezCastro 2018); por lo que hay un desfase en la integración del conocimiento generado desde hace más de una década.

El presente trabajo aborda el contexto histórico del conocimiento de Apocynaceae y cómo éste se ha consolidado hasta nuestros días en México, destacando al país como uno de sus centros de diversidad. Además, se presenta una actualización del número de géneros y especies de la familia en México, así como los cambios nomenclaturales más recientes. Se integra una clave taxonómica para determinar los géneros registrados en el país y se documenta su distribución geográfica por estado. Por último, se presentan algunas de las líneas de investigación que requieren mayor análisis e imágenes de taxones representativos.

\section{Materiales y métodos}

Para desarrollar el recuento histórico sobre el estado de arte de las Apocynaceae en México y la construcción de una línea temporal de descubrimientos, se tomaron en cuenta las fechas de publicación de todos los nombres aceptados actualmente y sus sinonimias. Se realizó la búsqueda de protólogos y de documentos históricos donde se ha hecho mención de las especies de Apocynaceae, como la Biblioteca Digital Mundial (2016), Biodiversity Heritage Library (2019), IPNI (2019) y Tropicos (2019). La información obtenida se organizó de acuerdo con la fecha de su publicación original y se realizó un histograma para observar los patrones temporales en que se describieron las especies, así como los taxónomos involucrados (Tabla 2, Figura 4). Cada uno de estos períodos es explicado para describir cómo se ha consolidado el conocimiento del grupo en el país.

Para la actualización del número de especies, su nomenclatura y su distribución, se realizó una búsqueda bibliográfica intensiva sobre el estudio de Apocynaceae en México, la cual constó de la revisión de diferentes trabajos taxonómicos, morfológicos y filogenéticos. De igual forma se apoyó en la revisión de ejemplares depositados en los herbarios nacionales e internacionales de CICY, ENCB, FCME, FEZA, G, HGOM, HNT, HUAP, HUMO, IBUG, IEB, MEXU, MO, NY, OAX, QMEX, SERO, UAMIZ y XAL (Thiers 2019); También se revisaron los herbarios virtuales del Portal de Datos Abiertos UNAM (2019), $\underline{\mathrm{HUH}}$ (2019), Global Plants Jstor (2019), Smithsonian National Museum of Natural History (2019), y bases de datos públicas como Naturalista (2019), Neotropical Key Kew (2019), The International Plant Names Index (2019) y Tropicos (2019). Asimismo, los autores han realizado desde el año 2000 a la fecha salidas al campo a diferentes puntos del país, lo que permitió recolectar especímenes y fotografías de las distintas especies de Apocynaceae. Con base en estos recursos mencionados, se construyó la clave de identificación de géneros para la familia (Material suplementario 1), la lista actualizada de especies, que incluye los taxones nativos e introducidos que cuentan con respaldo en las colecciones consultadas (Material suplementario 2). Para la actualización de los nombres y las combinaciones nuevas de los diferentes taxones del grupo, se siguieron la mayoría de las propuestas filogenéticas 

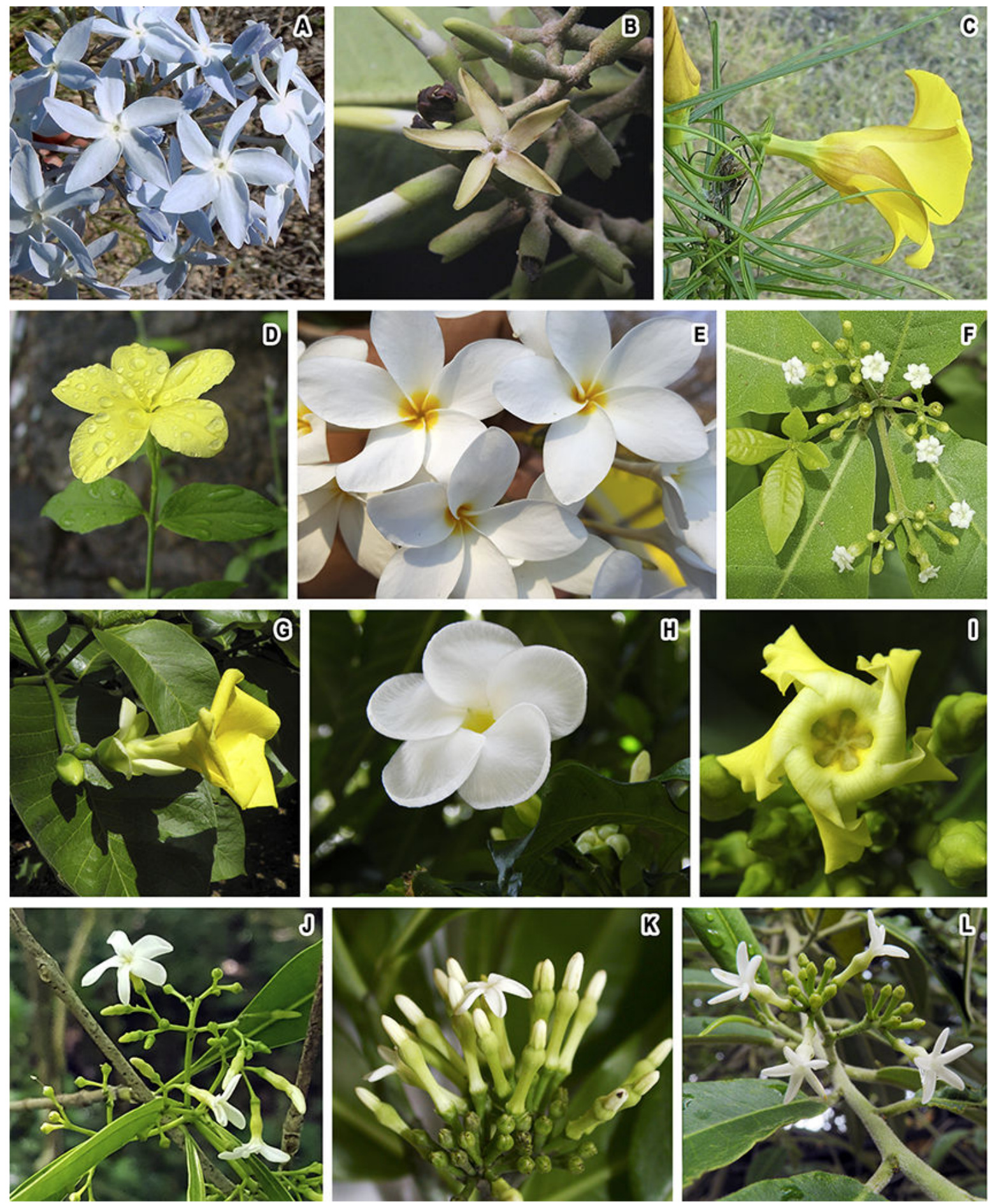

Figura 1. Algunos representantes de las rauvolfioides en México. A) Amsonia longiflora. B) Aspidosperma megalocarpon. C) Cascabela pinifolia. D) Haplophyton cimicidum. E) Plumeria rubra. F) Rauvolfia tetraphylla. G) Tabernaemontana glabra. H) T. litoralis. I) Thevetia ahouai. J) Tonduzia longifolia. K) Vallesia aurantiaca. L) V. conzattii. Créditos fotográficos: José Paulín (A), Allen Coombes (B), Francisco Morales (K), Abisaí García (L) 
(Simões et al. 2010, Fishbein et al. 2011, Liede-Schumann \& Meve 2008, 2013, Morillo 2015, Morales et al. 2017, McDonnell et al. 2018, do Espírito Santo et al. 2019) y revisiones taxonómicas recientes (Alvarado-Cárdenas \& Morales 2014, Lozada-Pérez \& Alvarado-Cárdenas 2015, Fishbein 2017, Morales \& Zamora 2017, Endress et al. 2018, Stevens 2018, Alvarado-Cárdenas et al. 2017, Alvarado-Cárdenas et al. 2019). Los cambios a nivel de género se discuten en el trabajo y se señalan en la lista de especies (Material suplementario 2).

Para la distribución se señala en las diferentes tablas la distribución conocida por estado, el endemismo y microendemismo (especies presentes en un solo estado). Con base en la búsqueda bibliográfica intensiva se registró la categoría de riesgo en la cual se han asignado las especies de la familia para el país. Las categorías de riesgo se señalan en la lista de especies (Material suplementario 2).

\section{Resultados}

Conocimiento histórico de Apocynaceae en México. Prelinneanos. El primer registro que se tiene del grupo en el país data de los restos encontrados en una cueva en Tehuacán, Puebla, México, de una inflorescencia de Plumeria rubra L. y endocarpos pétreos de Cascabela thevetia (L.) Lippold. Los restos encontrados datan de más de 6,000 años Antes de la Era Común (Smith 1967). Este descubrimiento resalta lo importante que era el grupo para los pueblos precolombinos, de hecho, muchas de sus especies están presentes y en uso por pueblos autóctonos de México (Juárez-Jaimes et al. 2007, Fernández-Brewer et al. 2008, Alvarado-Cárdenas et al. 2017).

Posterior a la conquista de México, el conocimiento de las plantas se enfocó en la recopilación del saber utilitario que se les daba a los recursos vegetales. Los trabajos del Códice Cruz-Badiano (De la Cruz 1552, Miranda \& Valdés 1964), Códice florentino (Sahagún 1577) y el Códice Voynich (Janick \& Tucker 2017) aportan los primeros documentos que ilustran las plantas, dan el nombre en náhuatl y los diferentes usos de Apocynaceae empleados por los pueblos precolombinos. Un libro fundamental es Historia Natural de la Nueva España del protomédico Francisco Hernández (1517-1587), en el cual se registra el uso de 21 especies de Apocynaceae. Este trabajo es una de las primeras aproximaciones al conocimiento taxonómico, ya que además de presentar los usos de los taxones, utilizó su nombre latino que después retomaría Linneo.

Linneanos y las grandes exploraciones. Linnaeus (1753) en su magna obra de Species Plantarum cita, en su Pentandria Digyna, 54 especies de Apocynaceae, de las cuales 10 están presentes de forma natural en México. Otro aporte lo proporciona Jacquin, quien era un aficionado Apocinólogo (Jacquin 1806), que trabajó principalmente en el Caribe,
Venezuela y Colombia (Jacquin 1760, Rowley 1984, Madriñán 2009), y que posteriormente sus especies descritas se recolectaron en México.

Los siguientes trabajos importantes son las exploraciones hechas por diferentes naturalistas, principalmente extranjeros, a lo largo de América y algunos enfocados en México. Las expediciones de A. von Humboldt y A. Bonpland (1803-1804), de H. Galeotti (1835-1843), del HSM. Sulphur (Hooker \& Arnott 1841, Bentham 1845), entre otras, marcaron una etapa de grandes descubrimientos en la biodiversidad. En este punto se describieron numerosas especies tanto para Apocynaceae como para la flora nacional. A la par de estas expediciones, diversos botánicos se involucraron en la descripción de especies y géneros nuevos de Apocynaceae para México. Ejemplo de ellos son AP. de Candolle (1844) que describió los géneros Haplophyton A. DC. y Laubertia A. DC., y J. Decaisne (1844) que describió numerosos géneros, seis de ellos presentes en México (Chtamalia Decne., Dictyanthus Decne., Orthosia Decne., Pherotrichis Decne. (Decaisne 1838), Polystemma Decne. y Tassadia Decne.), y quien además destaca como uno de los botánicos con más de una veintena de especies aceptadas y registradas en el país (Tabla 2). Se puede resaltar la expedición de la Nueva España (hoy México) por M. Sessé y M. Mociño, que representa la primera aportación en la diversidad nacional de Apocynaceae. En la Flora Mexicana (Sessé \& Mociño $\underline{1893}, \underline{1894)}$ se documentan un total de 13 géneros y 51 especies, muchas de ellas representaban taxones inéditos. Sin embargo, los infortunios de la época y la tardanza de la publicación resultaron en que muchas de las novedades taxonómicas fueron publicadas por otros botánicos (McVaugh 1969).

Un papel fundamental fue el de los colectores G. Andrieux y W.F. Karwinski, quienes aportaron importantes registros y descubrimientos botánicos, incluidas numerosas Apocynaceae. Andrieux no tenía una cantidad elevada de números de colecta, pero su trabajo fue destacado y revisado por botánicos renombrados de la época (e.g., A.P. de Candolle y Hemsley) e integradas en sus obras (Prodromus y Biologia Centrali-Americana) y colecciones de los herbarios (Sousa 1979). Otro de los colectores de gran relevancia para la flora nacional fue Karwinski. Este recolector visitó diferentes estados del país y proporcionó numerosas novedades taxonómicas (McVaugh 1980), entre las que se pueden señalar: Trichosacme lanata Zucc., género monotípico de Asclepiadoideae y endémico de México, descrita por Zuccarini en 1846.

Naturalistas y botánicos extranjeros. En inicios del siglo $\mathrm{XX}$, numerosos botánicos extranjeros describieron la biodiversidad vegetal de Estados Unidos de América y de México. Entre ellos se cuentan el destacado Asa Gray, 

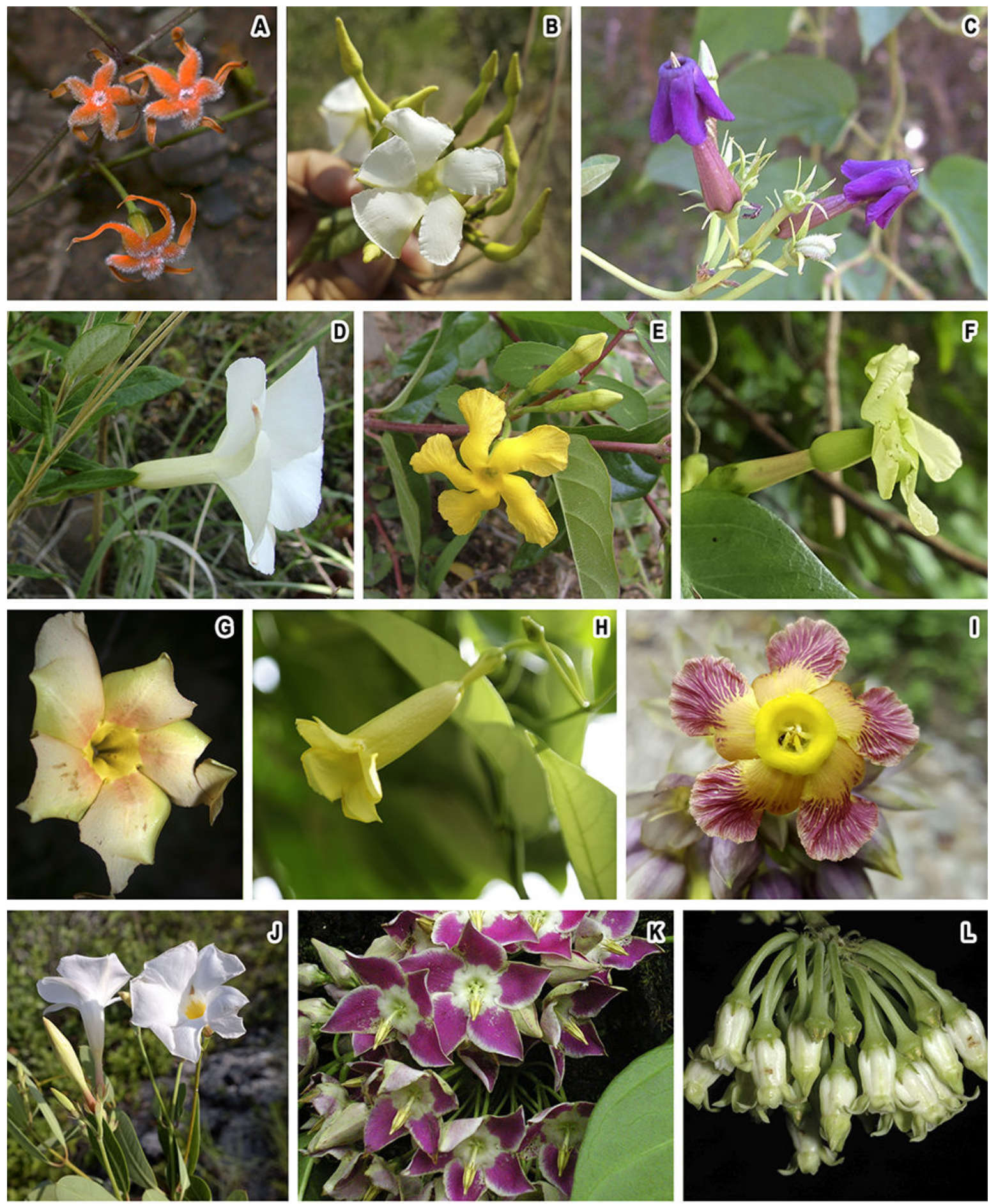

Figura 2. Algunos representantes de las apocynoides en México. A) Echites woodsonianus. B) E. yucatanensis. C) Laubertia contorta. D) Mandevilla hypoleuca. E) M. mexicana. F) Mesechites trifidus. G) Odontadenia semidygina. H) Pentalinon andrieuxii. I) Prestonia portobellensis. J) Rhabdadenia biflora. K) Thenardia floribunda. L) Thoreauea guerrerensis. Créditos fotográficos: Francisco Morales (A y G), Víctor Steinmann (L). 
J. Greenman y T. Brandegee, quienes aportaron más de una decena de especies de Apocynaceae para México (Tabla 2). A la par de las descripciones por los taxónomos, el trabajo de los colectores resultó fundamental en el conocimiento florístico. Entre ellos resaltan C. Pringle y C. Purpus con una importante actividad de trabajo de campo en varios estados del país y muchas de sus recolectas resultaron en novedades taxonómicas (Sousa 1969). En el periodo de 1882-1884, Pringle tuvo una prolífica actividad como colector con 2,700 números (Mauz 2011). El trabajo de Purpus fue documentado en una serie de publicaciones por Brandegee (Brandegee 1906, 1908, 1909) y posteriormente por Standley (1926) y, Sousa 1969). Entre los taxones descritos por Brandegee, destaca el género monotípico Microdactylon Brandegee (Brandegee 1908), ahora subordinado a Polystemma (Endress et al. 2018).

Paul C. Standley (1924) publicó la sección de Trees and Shrubs of Mexico, en donde se presenta el tratamiento de las Apocynaceae y Asclepiadaceae conocidas hasta el momento. Allí registró un total de 40 géneros (Apocynaceae $=17$, Asclepiadaceae $=23$ ) y 171 especies nativas $($ Apoc $=64$, Asclep $=107)$. Este trabajo representó la segunda aproximación al conocimiento de la diversidad del grupo para el país.

Especialistas botánicos. Los siguientes picos más importantes en el conocimiento de la familia, se enfocan en el trabajo de los especialistas de Apocynaceae para América, cuya actividad se ubica entre 1930 y 1950. Durante este periodo, Woodson proporciona los trabajos más completos sobre el conocimiento de ApocynaceaeAsclepiadaceae y una prolífica descripción de especies nuevas (Woodson 1928a, b $, 1932 \mathrm{a}, \underline{\mathrm{b}}, 1933,1934,1935$, $\underline{1938}, \underline{1939}, \underline{1941}, \underline{1945}$ ), de las cuales, 38 se encuentran como nombres aceptados en México (Tabla 2). También realizó algunos tratamientos regionales, como Flora of North America (Woodson 1938) y The apocynaceous flora of the Yucatan Peninsula (Woodson 1940). Igualmente, Marcel Pichon fue un taxónomo de gran importancia, contemporáneo de Woodson, que realizó una serie de trabajos fundamentales por sus análisis taxonómicos y las ilustraciones precisas (Pichon 1948a, $\underline{b}, \underline{c}, 1949,1950 \mathrm{a}, \underline{b}$ ); no obstante, él no describió alguna especie adicional.

La nueva exploración mexicana y los nuevos apocinólogos. Durante mediados del siglo XX a la fecha, se presentan los cambios más importantes en el grupo. Este período constituye un tiempo de integración de metodologías novedosas que han cambiado la sistemática de la familia. A finales de la década de los 50, en México surge un renovado interés por conocer y documentar los recursos vegetales. Entre ellos se encuentran los muestreos realizados por la Comisión de Dioscóreas (Gómez-Pompa 2016) y los esfuerzos de colecta para generar los listados florísticos (Gómez-Pompa \& Nevling 1970, Dávila-Aranda et al. 1993, Sosa \& Gómez-Pompa 1994) y las diferentes floras regionales. Esta serie de trabajos permitieron la descripción de numerosas especies nuevas y de registros nuevos. La familia o algunos de sus miembros se han revisado regionalmente en Flora de Baja California (Wiggins 1980), Flora de Guerrero (Diego-Pérez 2004, Juárez-Jaimes \& Ángeles-Trujillo 2013), Flora de la Península de Yucatán (Herbario CICY 2010-2020), Flora del Bajío y Regiones Adyacentes (Rzedowski \& Calderón de Rzedowski 1998), Flora del Valle de Tehuacán-Cuicatlán (Juárez-Jaimes \& Lozada-Pérez 2003, Alvarado-Cárdenas 2004), Flora Fanerogámica del Valle de México (Marroquín \& Calderón de Rzedowski 2001, Stevens 2001a), Flora Mesoamericana (Stevens \& Morales 2009), Flora y vegetación del desierto de Sonora (Shreve \& Wiggins 1964), así como en los estados de Hidalgo (Cervantes-Meza 2018, Cortez-Castro 2018), Morelos (González-Rocha \& Cerros-Tlatilpa 2015, Rodríguez-Morales 2015) y la región de Los Tuxtlas en Veracruz (Hernández-Barón 2017, Hernández-Barón et al. 2019).

A la par de estos tratamientos taxonómicos, principalmente a finales del siglo pasado (1980) a la fecha, se han realizado revisiones para algunos taxones mexicanos de Apocynaceae: Aspidosperma Mart. \& Zucc. (Morales \& Zamora 2017), Bruceholstia Morillo (Morillo 2015), Cascabela Raf. (Alvarado-Cárdenas \& Ochoterena 2007, Alvarado-Cárdenas et al. 2017), Cynanchum L. (Sundell 1981, Liede 1997, Liede \& Täuber 2002, Stevens 2005), Echites P. Browne (Morales 1997a, 2002b, Morales \& Williams 2004, Williams 2002b, 2004a), Fischeria DC. (Murphy 1986), Haplophyton (Williams 1995), Laubertia (Morales 2002a), Mandevilla Lindl. (Alvarado-Cárdenas \& Morales 2014, Lozada-Pérez \& Alvarado-Cárdenas 2015), Metalepis Griseb. (Morillo 1997), Metastelma R. Br. (Liede-Schumann \& Meve 2004), Odontadenia Benth. (Alvarado-Cárdenas 2016), Orthosia (Liede-Schumann \& Meve 2008, 2013), Pattalias S. Watson (Fishbein \& Stevens 2005, Fishbein 2017), Pentalinon Voigt (Hansen \& Wunderlin 1986), Pinochia M.E. Endress \& B.F. Hansen (Endress \& Hansen 2007), Prestonia R. Br. (Morales 1996, 1997a,b), Rotundanthus Morillo (Morillo 2015), Suberogerens Morillo (Morillo 2015), Tabernaemontana L. (Alvarado-Cárdenas et al. 2019), Thenardia Kunth (Williams 1998), Thevetia L. (Alvarado-Cárdenas \& Ochoterena 2007), Thoreauea J.K. Williams (Williams 2002a, Morales 2005), Vailia Rusby (Stevens 2018) y Vulcanoa Morillo (Morillo 2015) y algunos no publicados para las apocynoides (Williams 1999), Dictyanthus (González-Martínez 2019), Marsdenia R. Br. (JuárezJaimes 1998) y Pherotrichis (Lozada-Pérez 2003). 

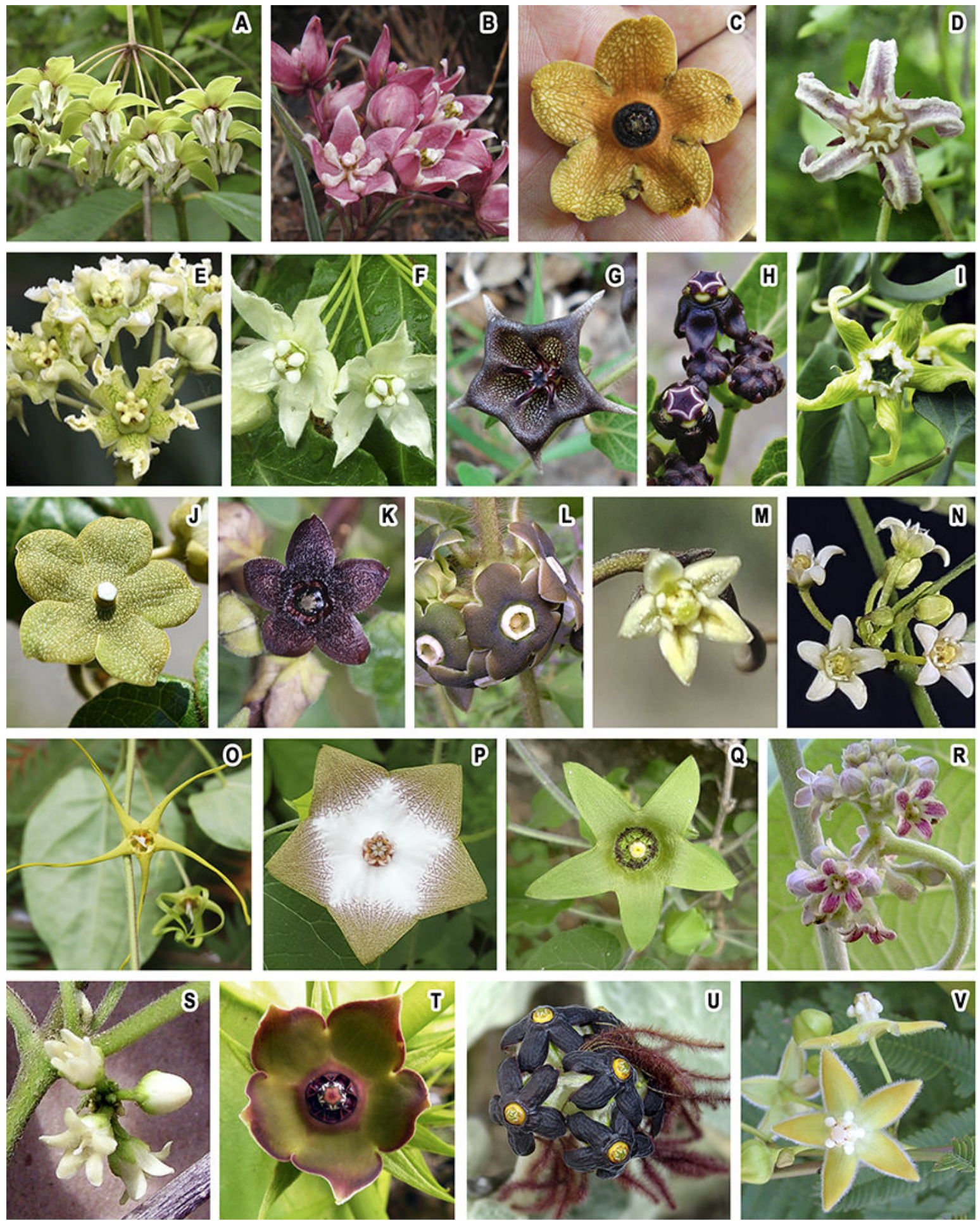

Figura 3. Algunos representantes de Asclepiadoideae en México. A) Asclepias auriculata. B) A. circinalis. C) Bruceholstia magnifolia. D) Cynanchum ligulatum. E) Fischeria scandens. F) Funastrum pannosum. G) Dictyanthus reticulatus. H) Gonolobus niger. I) G. pectinatus. J) Matelea velutina. K) M. tezcatlipocantha. L) Macroscepis pleistantha. M) Metastelma lanceolata. N) Orthosia angustifolia. O) Oxypetalum cordifolium. P) Polystemma guatemalense. Q) Polystemma viridiflorum. R) Ruehssia mexicana (antes Marsdenia mexicana) S) R. coulteri (antes Marsdenia coulteri). T) Suberogerens cyclophylla. U) Trichosacme lanata. V) Vailia anomala. Créditos fotográficos: Matus Hernández (E), Leccinium García (H y T), Allen Coombes (Q) 
En el periodo de 1958-2019, se han descrito alrededor de 80 especies, casi un cuarto de la diversidad conocida para la familia. Asimismo, se consolidan diferentes especialistas como W.D. Stevens, M. Fishbein, J. Williams, V. JuárezJaimes, J.F. Morales, L. Lozada-Pérez y L.O. AlvaradoCárdenas. Cabe destacar la actividad de W.D. Stevens, quien es el segundo sistemático con más especies descritas de Apocynaceae en México (Tabla 2) y que ha aportado numerosas revisiones taxonómicas para mejorar la comprensión de la familia (Stevens 1975, 1988, 1999, 2000, 2001a, b, 2005, Stevens \& Morales 2009).

Solo incluyendo a las especies aceptadas, un total de 104 botánicos (110 incluyendo sinónimos) han descrito alguna de las especies de Apocynaceae registradas para México. De esta centena, 14 taxónomos han descrito 10 o más especies, aportando más del $50 \%$ (263 spp.) de la diversidad conocida para el país (Tabla 2). Los taxónomos mexicanos están representados por 12 personas, de los cuales V. Juárez-Jaimes, L. Lozada-Pérez y L. AlvaradoCárdenas son los especialistas con más de 10 especies descritas y con diferentes aportes sistemáticos (JuárezJaimes \& Lozada-Pérez 2003, Alvarado-Cárdenas 2004, 2007, Alvarado-Cárdenas \& Juárez-Jaimes 2011, 2012 Juárez-Jaimes et al. 2007, Juárez-Jaimes \& AlvaradoCárdenas 2010, Lozada-Pérez \& Diego-Pérez 2012, Juárez-
Jaimes \& Lozada-Pérez 2015, Lozada-Pérez \& AlvaradoCárdenas 2015, 2016).

Además de la descripción de especies y revisiones, a mediados de 1990 se comenzaron a aplicar herramientas nuevas para evaluar la sistemática del grupo. Los análisis filogenéticos, principalmente con datos moleculares, permitieron reevaluar la clasificación a nivel de familia (Endress et al. 1996, Sennblad \& Bremer 1996, 2000, Potgieter \& Albert 2001, Yang et al. 2016), subfamilia (Simões et al. 2007, Livshultz et al. 2007), tribus (Sennblad et al. 1998, Simões et al. 2004, 2010) y géneros (Simões et al. 2006, Alvarado-Cárdenas \& Ochoterena 2007, Krings et al. 2008, Fishbein et al. 2011, Morales et al. 2017). De los cerca de 70 análisis filogenéticos que se han desarrollado para la familia, se han integrado más de 30 géneros y 150 especies registradas para el país. Otras herramientas actualmente empleadas son los sistemas de información geográfica y el modelado de nicho, los cuales han permitido entender mejor los patrones de distribución de las especies y de su diversidad en el país. Los trabajos más recientes presentan más argumentos para sugerir categorías de riesgo para las especies y su potencial conservación (AlvaradoCárdenas et al. 2017, 2019).

A la par del conocimiento sistemático, otros rubros se han abordado en el grupo, pero no con tanta atención. Entre

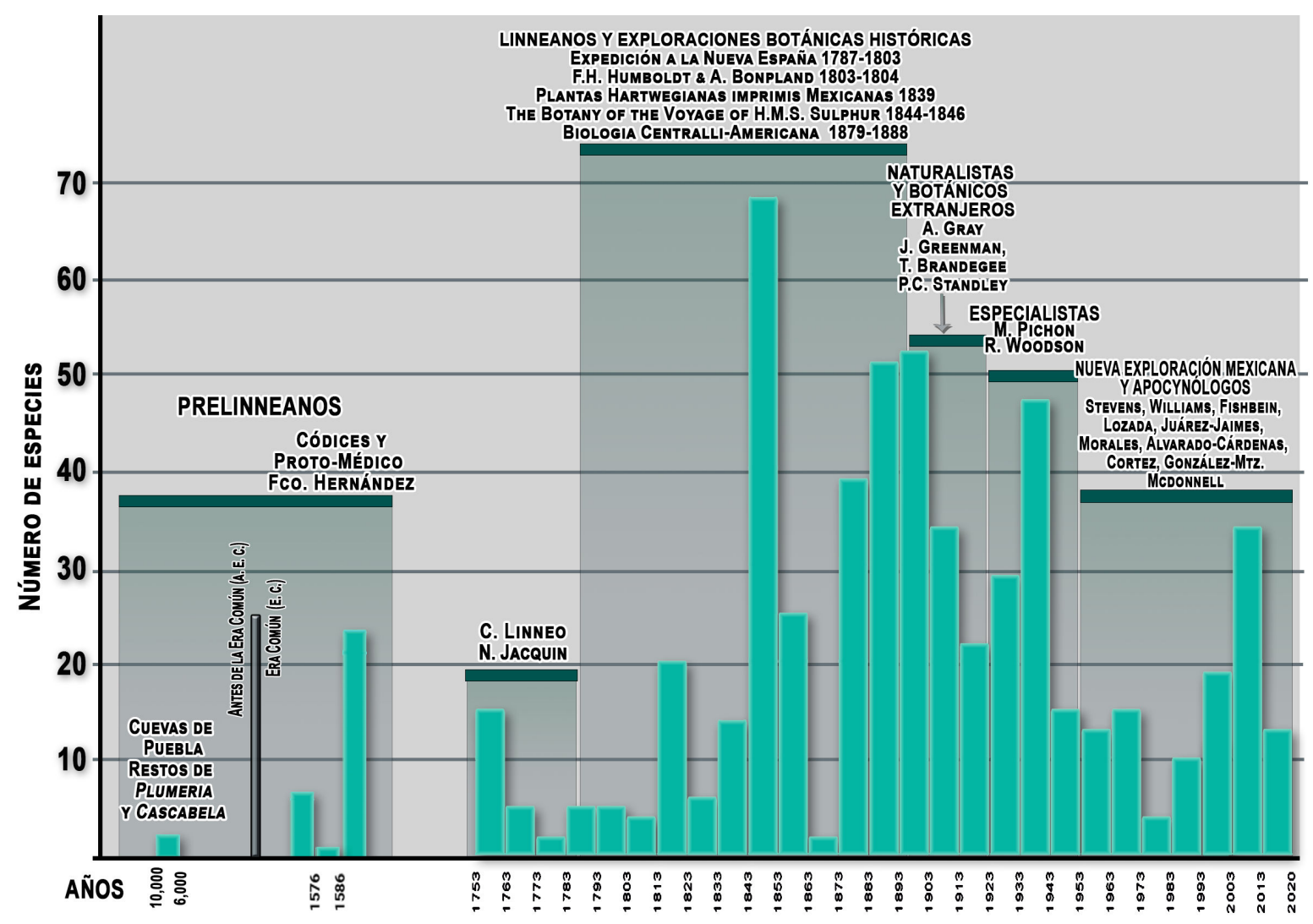

Figura 4. Relación histórica del conocimiento de la diversidad de Apocynaceae en México. 
Apocynaceae en México

Tabla 2. Relación de algunos sistemáticos que han descrito especies aceptadas de Apocynaceae presentes en México

\begin{tabular}{lclc}
\hline Sistemáticos con más de 10 spp aceptadas & No. especies & Sistemáticos mexicanos con especies aceptadas No. especies \\
\hline R.E. Woodson & 38 & V. Juárez-Jaimes & 14 \\
W.D. Stevens & 35 & L.O. Alvarado-Cárdenas & 14 \\
A. Gray & 30 & L. Lozada-Pérez & 12 \\
J. Decaisne & 25 & E.B. Cortez & 2 \\
T. Brandegee & 19 & N. Diego & 2 \\
P.C. Standley & 18 & J.M. Mociño & 2 \\
V. Juárez-Jaimes & 14 & A. Campos & 1 \\
L.O. Alvarado-Cárdenas & 14 & C.A. González-Martínez & 1 \\
J. Torrey & 13 & S. Islas-Hernández & 1 \\
C. Linneo & 12 & K.G. Maya & 1 \\
L. Lozada-Pérez & 12 & A. Saynes & 1 \\
J. Donnell-Smith & 11 & V. Saynes & 1 \\
J.M. Greenman & 11 & J. Soto & 1 \\
G. Bentham & 11 & & \\
\hline
\end{tabular}

ellos se pueden señalar trabajos de índole fitoquímica con especies de Asclepias L. (Domínguez et al. 1958, Fonseca et al. 1991, Navarro-García et al. 2003, Rascón-Valenzuela et al. 2015), Cascabela (Ramos-Silva et al. 2017), Haplophyton (Mroue et al. 1993, Llanos-Romero et al. 2014) y Tabernaemontana (Krengel et al. 2016, 2019). Los de conocimiento etnobotánico (Heinrich et al. 1992, GarcíaAlvarado et al. 2001, Fernández-Brewer et al. 2008), de polinización (Kunze 1999, Liede 1994, Ollerton et al. 2018), así como de ecología de frutos y semillas (CoatesEstrada et al. 1993, Bebawi et al. 2017).

Diversidad actual de Apocynaceae de México. Las Apocynaceae están representadas por 52 géneros y 418 especies nativas en México, ubicadas en tres grupos rauvolfioides, apocynoides y Asclepiadoideae (Tabla 1, $\underline{3}$, 4). Las rauvolfioides están representadas por 11 géneros y 46 especies (Figura 1), seguido de las apocynoides con 14 géneros y 57 especies (Figura 2). La subfamilia Asclepiadoideae es la más diversa, con 27 géneros y 315 especies (Figura 3), y presentó nuevas adiciones a nivel genérico con la segregación de diferentes taxones de Matelea Aubl. (e.g., Brucehosltia, Suberogerens, Vulcanoa, entre otros). Mientras que en apocynoides y rauvolfioides varios taxones se subordinaron a distintos géneros, pero tuvieron la incorporación de nuevas especies (Material suplementario 1,2).

De las 25 tribus conocidas para el mundo, 12 están presentes en México. Las apocynoides presentan cinco de las nueve tribus reconocidas, seguidas de las rauvolfioides con 5 de 11 y Asclepiadoideae con 2 de 5 . Los géneros con mayor diversidad son Asclepias (72 spp.), Matelea (64 spp.), Gonolobus Michx. (38 spp.), Ruehssia H. Karst. ex Schltdl. (36 spp.), Metastelma (25 spp.) y Mandevilla (24 spp.), que en conjunto aportan más del $50 \%$ de la diversidad de la familia (Tabla 4). A su vez, estos taxones son los que tienen el mayor endemismo (40\% o mayor), junto con Dictyanthus (87.5\% endemismo), Tabernaemontana (47 \% endemismo), Polystemma y Cascabela (50 \% endemismo cada uno) (Tabla 3). Con estas cifras, la familia se ubica a nivel específico en el lugar número 12 a nivel nacional, mientras que a nivel genérico ocupa el octavo lugar.

Se encontraron al menos 19 géneros correspondientes a 24 especies cultivadas, de las cuales Asclepiadoideae presentó el mayor número de géneros, con ocho de ellos (Araujia Brot., Calotropis R. Br., Ceropegia L., Hoya R. Br., Huernia R. Br., Orbea Haw., Pseudolithos P.R.O. Bally, Stapelia L., Stephanotis Thouars), seguido de las Apocynoides con cinco (Adenium Roem. \& Schult., Beaumontia Wall., Nerium L., Pachypodium Lindl., Trachelospermum Lem.), rauvolfioides con cuatro (Allamanda L., Carissa L., Catharanthus G. Don, Vinca L.) y Periplocoideae con Cryptostegia R. Br. Algunos de estos géneros solo se conocen de cultivo o colecciones vivas y no hay ejemplares de herbario que respalden su presencia en el país. En conjunto con los taxones nativos, el total de géneros es de 71 y 442 especies. En el Material suplementario 1 se presenta una clave de identificación para los géneros nativos y cultivados registrados en el país. 
Alvarado-Cárdenas et al. / Botanical Sciences 98(2): 393-416. 2020

Tabla 3. Riqueza y endemismos de los géneros de Apocynaceae en México. Los asteriscos refieren a los géneros endémicos al país.

\begin{tabular}{|c|c|c|c|c|}
\hline Géneros & No. especies en México & No. endémicas (\%) & No. microendémicas (\%) & No. de estados en que está presente \\
\hline 1 Amsonia & 4 & 0 & 0 & 4 \\
\hline 2 Aросупит & 3 & 0 & 0 & 7 \\
\hline 3 Asclepias & 72 & $36(50.6)$ & $7(9.5)$ & 32 \\
\hline 4 Aspidosperma & 2 & 0 & 0 & 8 \\
\hline 5 Bruceholstia & 1 & 0 & 0 & 3 \\
\hline 6 Cameraria & 1 & 0 & 0 & 5 \\
\hline 7 Cascabela & 6 & $3(50)$ & 0 & 29 \\
\hline 8 Chthamalia & 7 & $5(71.4)$ & 0 & 15 \\
\hline 9 Cynanchum & 8 & $3(37.9)$ & 0 & 31 \\
\hline 10 Dictyanthus & 16 & $14(87.5)$ & $5(31)$ & 22 \\
\hline 11 Echites & 8 & $1(12.5)$ & 0 & 21 \\
\hline 12 Fischeria & 1 & 0 & 0 & 6 \\
\hline 13 Forsteronia & 3 & 0 & 0 & 7 \\
\hline 14 Funastrum & 13 & $3(23)$ & 0 & 32 \\
\hline 15 Gonolobus & 38 & $18(48.3)$ & $7(18.5)$ & 30 \\
\hline 16 Haplophyton & 2 & 0 & 0 & 14 \\
\hline 17 Himantostemma & 1 & 0 & 0 & 3 \\
\hline 18 Jobinia & 1 & 0 & 0 & 1 \\
\hline 19 Laubertia & 1 & $1(100)$ & 0 & 10 \\
\hline 20 Macroscepis & 2 & 0 & 0 & 13 \\
\hline 21 Mandevilla & 24 & $12(50)$ & $4(16)$ & 30 \\
\hline 22 Matelea & 64 & $42(65.6)$ & $16(25)$ & 32 \\
\hline 23 Mesechites & 1 & 0 & 0 & 6 \\
\hline 24 Metalepis & 1 & 0 & 0 & 3 \\
\hline 25 Metastelma & 25 & $12(48)$ & $3(12)$ & 27 \\
\hline 26 Odontadenia & 1 & 0 & 0 & 4 \\
\hline 27 Orthosia & 9 & $5(55)$ & $2(22.2)$ & 19 \\
\hline 28 Oxypetalum & 1 & 0 & 0 & 12 \\
\hline 29 Pattalias & 2 & 0 & 0 & 10 \\
\hline 30 Pentalinon & 1 & 0 & 0 & 13 \\
\hline 31 Pherotrichis & 4 & $2(50)$ & 0 & 9 \\
\hline 32 Pinochia & 1 & 0 & 0 & 3 \\
\hline 33 Plumeria & 2 & 0 & 0 & 30 \\
\hline 34 Polystemma & 6 & $3(50)$ & $1(20)$ & 16 \\
\hline 35 Prestonia & 5 & $1(20)$ & 0 & 16 \\
\hline 36 Prosthecidiscus & 1 & 0 & 0 & 6 \\
\hline 37 Rauvolfia & 2 & 0 & 0 & 20 \\
\hline 38 Rhabdadenia & 1 & 0 & 0 & 6 \\
\hline 39 Rotundanthus & 1 & 0 & 0 & 1 \\
\hline 40 Ruehssia & 36 & $21(58.3)$ & $11(30.5)$ & 27 \\
\hline 41 Suberogerens* & 1 & $1(100)$ & 0 & 8 \\
\hline 42 Tabernaemontana & 17 & $8(47)$ & $5(29.4)$ & 25 \\
\hline 43 Tassadia & 1 & 0 & 0 & 3 \\
\hline 44 Thenardia & 3 & $2(66.6)$ & 0 & 9 \\
\hline 45 Thevetia & 1 & 0 & 0 & 17 \\
\hline 46 Thoreauea* & 3 & $3(100)$ & $2(66.6)$ & 3 \\
\hline 47 Tintinnabularia & 2 & $1(50)$ & 0 & 3 \\
\hline 48 Tonduzia & 1 & 0 & 0 & 11 \\
\hline 49 Trichosacme* & 1 & $1(100)$ & 0 & 3 \\
\hline 50 Vailia & 1 & 0 & 0 & 17 \\
\hline 51 Vallesia & 8 & $5(62.5)$ & $4(50)$ & 23 \\
\hline 52 Vulcanoa & 1 & 0 & 0 & 1 \\
\hline Total & 418 & $204(48.8)$ & $67(15)$ & \\
\hline
\end{tabular}


Distribución y endemismo de Apocynaceae de México. Los estados con 100 o más especies nativas son Oaxaca (182 spp.), Chiapas (159 spp.), Veracruz (125 spp.), Guerrero (118 spp.) y Jalisco (100 spp.). Los estados de Aguascalientes, Baja California y Tlaxcala presentan la menor diversidad con menos de 26 especies cada uno (Tabla 4). Las entidades con más especies endémicas son Oaxaca (85 spp.), Guerrero (55 spp.), Jalisco (53 spp.), Michoacán (49 spp.), Puebla (44 spp.) y México (38 spp.).

Tabla 4. Riqueza y endemismo de especies de Apocynaceae en cada uno de los 32 estados de México. En cada columna se resalta en negritas los estados con mayor riqueza

\begin{tabular}{|c|c|c|c|c|}
\hline Estados & Géneros & Especies & Endémicas & Microendémicas \\
\hline AGS & 10 & 24 & 7 & 0 \\
\hline $\mathrm{BCN}$ & 10 & 20 & 3 & 1 \\
\hline BCS & 14 & 25 & 10 & 5 \\
\hline CAM & 27 & 55 & 3 & 0 \\
\hline CDMX & 14 & 29 & 18 & 0 \\
\hline CHIS & 43 & 159 & 26 & 7 \\
\hline СНIH & 21 & 73 & 26 & 2 \\
\hline СОАН & 14 & 49 & 11 & 1 \\
\hline COL & 22 & 38 & 20 & 2 \\
\hline DGO & 14 & 54 & 18 & 1 \\
\hline GTO & 14 & 52 & 27 & 0 \\
\hline GRO & 33 & 118 & 55 & 3 \\
\hline HGO & 18 & 61 & 28 & 2 \\
\hline JAL & 28 & 100 & 53 & 7 \\
\hline MEX & 21 & 68 & 38 & 0 \\
\hline МICH & 27 & 96 & 49 & 3 \\
\hline MOR & 24 & 63 & 29 & 0 \\
\hline NAY & 22 & 58 & 27 & 2 \\
\hline NLE & 15 & 53 & 14 & 1 \\
\hline OAX & 40 & 182 & 86 & 17 \\
\hline PUE & 26 & 93 & 44 & 0 \\
\hline QRO & 21 & 62 & 25 & 0 \\
\hline QROO & 25 & 53 & 4 & 0 \\
\hline SLP & 23 & 71 & 27 & 1 \\
\hline SIN & 21 & 61 & 30 & 1 \\
\hline SON & 23 & 93 & 37 & 3 \\
\hline TAB & 26 & 48 & 1 & 0 \\
\hline TAMS & 21 & 63 & 16 & 2 \\
\hline TLAX & 9 & 14 & 9 & 0 \\
\hline VER & 40 & 125 & 32 & 4 \\
\hline YUC & 25 & 57 & 8 & 2 \\
\hline ZAC & 14 & 35 & 14 & 0 \\
\hline
\end{tabular}

Por su parte, el endemismo a nivel específico es del $48.8 \%$ (204 spp.) y a nivel genérico de $7.6 \%$ (Suberogerens (1 sp.), Thoreauea (3 spp.) y Trichosacme
(1 sp.)). Es importante señalar que, de los 32 estados de la república, solo 20 presentan microendémismos, de los cuales Oaxaca destaca con 17 especies, seguido de Chiapas y Jalisco con siete, Baja California Sur con cinco y Veracruz con cuatro (Tabla 4). A nivel genérico, Suberogerens es el taxón de más amplia distribución registrado en ocho estados, mientras que el resto se restringen a tres entidades cada uno

(Tabla 4).

Conservación de Apocynaceae de México. De las especies registradas para el país, 151 presentan alguna categoría de

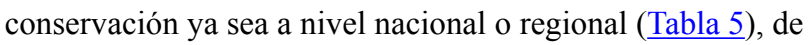
las cuales solo dos especies se han integrado a la Norma Oficial Mexicana NOM-059 (SEMARNAT 2010a, b), Asclepias mcvaughii Woodson y Vallesia spectabilis E. Mey. ex J.F. Morales que están bajo la categoría de protección especial (Pr). Las otras 149 especies (60 endémicas) se han asignado alguna de las categorías de la IUCN, a partir de las publicaciones de especies nuevas, las revisiones de géneros (Alvarado-Cárdenas et al. 2017 2019, González-Martínez 2019), así como de los tratamientos taxonómicos de la Península de Yucatán (Herbario CICY 2010-2020), Flora del Bajío y Regiones Adyacentes (Rzedowski \& Calderón de Rzedowski 1998), Hidalgo (Cervantes-Meza 2018, Cortez-Castro 2018) y Guerrero (Juárez-Jaimes \& Ángeles-Trujillo 2013). Destacan las Asclepiadoideae con más especies asignadas (93 spp.), seguidas de rauvolfioides (33 spp.) y apocynoides (25 spp.). Las especies endémicas de Tabernaemontana mixtecana L.O. Alvarado \& Juárez-Jaimes y T. stenoptera (Leeuwenb.) A.O. Simões \& M.E. Endress se asignaron a la categoría de extinta en su hábitat (Alvarado-Cárdenas \& Juárez-Jaimes 2012, Alvarado-Cárdenas et al. 2019).

\section{Discusión}

Las Apocynaceae son un grupo de importante diversidad específica y endémica para la flora mexicana. La construcción de este conocimiento ha pasado por etapas de importantes descubrimientos, como de pobre atención al grupo (Figura 4). Las herramientas nuevas de análisis, así como el trabajo de personas interesadas en la familia han resaltado sus más de 400 especies como un elemento conspicuo de la vegetación de la República Mexicana. Por lo que una actualización del conocimiento taxonómico, aquí presentada, era necesaria para integrar todos los cambios desarrollados en ya más de una década.

Diversidad y sistemática de Apocynaceae de México. Juárez-Jaimes et al. (2007) reportaron 51 géneros y 385 especies, desde entonces han ocurrido cambios en la circunscripción de los taxones, derivados de las inferencias filogenéticas, las revisiones y la descripción de numerosas 
Tabla 5. Relación de especies totales y endémicas de Apocynaceae asignadas a alguna categoría de conservación de acuerdo con la IUCN o la NOM-059.

\begin{tabular}{|c|c|c|c|c|c|c|c|c|c|c|}
\hline \multicolumn{2}{|l|}{ Taxón } & \multicolumn{9}{|c|}{ Categoría de riesgo } \\
\hline & & DD & LC & NT & $\mathbf{V U}$ & $\mathbf{E N}$ & CR & $\mathbf{E X}$ & PR & Total \\
\hline \multirow[t]{2}{*}{ Apocynaceae } & Total & 9 & 72 & 4 & 12 & 28 & 22 & 2 & 2 & 151 \\
\hline & Endémicas & 4 & 8 & 2 & 7 & 23 & 14 & 2 & 2 & 62 \\
\hline \multirow[t]{2}{*}{ Apocynoides } & Totales & & 15 & & & 1 & 9 & & & 25 \\
\hline & Endémicas & & & & & 1 & 2 & & & 3 \\
\hline \multirow[t]{2}{*}{ Asclepiadoideae } & Totales & 9 & 39 & 3 & 7 & 22 & 12 & & 1 & 93 \\
\hline & Endémicas & 4 & 7 & 1 & 5 & 18 & 11 & & 1 & 47 \\
\hline \multirow[t]{2}{*}{ Rauvolfioides } & Totales & & 18 & 1 & 5 & 5 & 1 & 2 & 1 & 33 \\
\hline & Endémicas & & 1 & 1 & 2 & 4 & 1 & 2 & 1 & 12 \\
\hline
\end{tabular}

Categorías IUCN: DD: Datos Insuficientes, LC: Preocupación menor, NT: Casi amenazado, VU: Vulnerable, EN: En peligro, CR: Peligro crítico, EX: Extinto.

Categoría NOM-059: PR: sujetas a protección especial

especies. Casi 10 años después, en el trabajo de Villaseñor $\underline{(2016)}$ se reconocen 58 géneros y 418 especies, pero incluye varios sinónimos genéricos y específicos (e.g., Allotoonia J.F. Morales \& J.K. Williams ahora Echites, Telosiphonia (Woodson) Henrickson ahora Mandevilla, Alstonia longifolia (A. DC.) Pichon ahora Tonduzia longifolia (A. DC.) Markgr., entre otros), por lo que las cifras difieren tanto en el número como en la identidad de los taxones que aquí se proporciona (52 géneros y 418 especies nativas, Tabla 3). Aunque la cantidad de géneros es casi la misma que la reportada en Juárez-Jaimes et al. (2007), han ocurrido numerosos cambios con la subordinación o el reconocimiento de diferentes taxones en cada una de las subfamilias presentes en el país.

En el caso de las rauvolfioides, el género Stemmadenia Benth. fue subordinado a sinonimia. Los miembros de este taxón se distinguían por las flores relativamente grandes y amarillas, con una corona corolina laminar y brácteas subtendiendo los sépalos (Morales \& Méndez 2005). La inferencia filogenética, basada en datos moleculares y morfológicos (Simões et al. 2007, 2010), mostró que las especies de Stemmadenia analizadas están anidadas dentro del clado pantropical Tabernaemontana, por lo que fueron sinonimizadas de este último. Actualmente, Tabernaemontana es uno de los géneros más diversos en el país con 17 especies nativas (Alvarado-Cárdenas et al. 2019, Figura 1).

Para las apocynoides, los géneros Allotoonia y Fernaldia Woodson fueron segregados de Echites a partir de sus distintos caracteres florales (Woodson 1932b, Morales \& Williams 2004); sin embargo, evidencia filogenética demostró que Allotoonia es un grupo parafilético con respecto a Echites y, de igual forma, Fernaldia quedó anidada dentro de Echites (Simões et al. 2007, Morales et al. 2017). Para mantener una estabilidad taxonómica Fernaldia y Allotoonia se consideran sinónimos de Echites (Figura 2, Material suplementario 1,2).

Las Asclepiadoideae presentaron cambios notables con el reconocimiento de los géneros Bruceholstia, Chthamalia, Himantostemma A. Gray, Rotundanthus, Suberogerens y Vulcanoa, los cuales fueron segregados de Matelea con base en los resultados de análisis filogenéticos y morfológicos (Morillo 2015, Endress et al. 2018, McDonnell et al. 2018). Matelea es un taxón de una circunscripción complicada, en el cual Woodson (1941) subordinó alrededor de 30 géneros como sinónimos y reconoció 13 subgéneros, y el número de especies que lo componen puede incluir más de 200 (Krings 2011, McDonnell et al. 2018). Se ha sugerido que el estudio detallado del mismo podría reducir su diversidad a no más de 30 especies (Stevens \& Morales 2009, Morillo 2012, 2015, Krings \& Morillo 2015, Lozada-Pérez \& AlvaradoCárdenas 2016). Aunque se está lejos de resolver la sistemática de este grupo, Stevens (1998), Krings (2011), Morillo (2015), McDonnell et al. (2018) y GonzálezMartínez (2019), han realizado análisis filogenéticos que han contribuido en la resolución de la problemática de Matelea y grupos afines. El último tratamiento taxonómico para los géneros de la familia a nivel mundial (Endress et al. 2018) recopila y describe los cambios propuestos para este y otros taxones. Algunos de dichos cambios se han integrado al presente trabajo y se han realizado las combinaciones correspondientes, así como su integración en la clave para los géneros presentes en el país (Material suplementario 1).

Otro cambio importante en los nombres es el de Blepharodon mucronatum (Schltdl.) Decne. Stevens (2018) 
propuso su transferencia a Vailia basado en las observaciones de esta y de otras especies de Blepharodon Decne. Observaciones previas sobre esta especie señalan que la estructura floral no coincide con el género (Morillo 1976). Asimismo, los resultados de las filogenias moleculares muestran que Blepharodon no es monofilético (Liede-Schumann et al. 2005, Rapini et al. 2006), por lo que el cambio realizado por Stevens (2018) muestra congruencia con la información actual.

Un caso reciente es la resurrección del género Ruehssia (Do Espírito Santo et al. 2019), el cual estaba subordinado a Marsdenia. El resultado del análisis filogenético de do Espírito Santo et al. (2019) recobra a las especies de Marsdenia en diferentes clados y relacionados con diferentes géneros. Un grupo asociado a la especie tipo

(M. tinctoria R.Br.) se restringe principalmente a Asia, mientras que las correspondientes a Ruehssia se ubican desde México hasta Sudamérica. Dichos autores realizaron los cambios nomenclaturales para las especies de Brasil y actualizaron la diagnosis genérica. Apoyado en este análisis, aquí se proponen las combinaciones correspondientes a las especies mexicanas.

En cuanto a las especies introducidas podemos notar que hay numerosos taxones (19 géneros y 24 especies), lo cual habla del valor ornamental del grupo. No obstante, también es importante tener en cuenta que algunas de estas especies exóticas son catalogadas como invasoras en otros países y con importantes efectos en la biodiversidad; por ejemplo, Araujia sericifera Brot. (Coombs \& Peter 2010), Calotropis procera (Aiton) W.T. Aiton (Menge et al. 2017) y Cryptostegia grandiflora R. Br. (Grice 1997). Un monitoreo de las especies introducidas aquí reportadas para saber su distribución y capacidad de dispersión es necesario para sugerir recomendaciones para su potencial control. De igual manera, el trabajo de divulgación es deseable para fomentar el cultivo de plantas nativas con potencial ornamental y económico, como algunos miembros de Asclepias, Cascabela, Gonolobus, Mandevilla, entre otros, los cuales presentan un importante endemismo y microendemismo. Un ejemplo a seguir son los esfuerzos realizados para el cultivo de especies de Asclepias en Estados Unidos, como el The Milkweed Zoo (2019) y varias páginas en internet que muestran especies nativas como In Defense of Plants $\underline{(2019)}$.

La integración del conocimiento actual de la sistemática del grupo es fundamental para poder tener una mejor comprensión de la biodiversidad de un país. Asimismo, la revisión constante de las colecciones biológicas juega un papel preponderante en generación y actualización de la información. Esto ha permitido desarrollar el listado de las especies conocidas para el país y su distribución (Tablas 3 , 4), así como la primera clave enfocada para los géneros mexicanos (Material suplementario 1). Esta herramienta sintetiza la enorme información recabada desde la lista de Juárez-Jaimes et al. (2007) a la fecha.

El estudio renovado de la familia Apocynaceae ha incrementado el número de trabajos que intentan resolver las relaciones filogenéticas entre los géneros y dentro de los mismos, lo que ha redituado en una mejor clasificación del grupo. Los análisis morfológicos bajo un marco filogenético han sugerido patrones de homología y evolución de diferentes estructuras vegetativas y florales, como las diferentes morfologías de las coronas ginostegiales del grupo (Endress \& Bruyns 2000, Fishbein 2001, Kunze 2005, Fishbein et al. 2018). Sin embargo, hace falta mucho trabajo para conocer el desarrollo de los apéndices florales en grupos de morfología floral complicada como las Asclepiadoideae. La integración de taxones mexicanos a las filogenias podrá repercutir en la resolución de la sistemática de grupos como las Gonolobineae, con una importante representación en el país.

La revisión taxonómica de los diferentes grupos ya sea a nivel de géneros o de floras regionales, está lejos de concluirse y de conocer de manera precisa la distribución y descripción de los taxones. De todos los tratamientos realizados para la familia en el país, se han abordado el $44 \%$ de los géneros conocidos. En particular, Asclepiadoideae requiere más atención, pues presenta la mayor diversidad y solo el $33 \%$ de sus géneros se ha abordado recientemente. Actualmente, se cuenta con tres especialistas a nivel nacional (Tabla 2) y al menos otros cuatro extranjeros que han realizado trabajo para la flora de la familia en México. Un reto difícil para la sistemática, en la perspectiva global, es la captación de más taxónomos y el reconocimiento de su labor (Wandersee \& Schussler 1999, Villaseñor 2015). No obstante, el trabajo de personas interesadas en la familia se está desarrollando (McDonnell et al. 2015, 2018, McDonnell \& Fishbein 2016, HernándezBarón 2017, Cortez et al. 2018, González-Martínez et al. $\underline{2019}$ ), pero es necesaria la continuidad e integración de más profesionistas taxónomos.

Distribución y endemismo. Los resultados aquí presentados corroboran lo observado en el trabajo de Juárez-Jaimes et al. (2007). Los estados con mayor número de especies de Apocynaceae son Oaxaca y Chiapas y las entidades asociadas a las vertientes del país, esto reafirma la afinidad tropical del grupo. Asimismo, se revalidan Asclepias, Matelea, Gonolobus y Ruehssia como los géneros con más especies y los que más amplia distribución presentan. Es muy probable que se reporten otras novedades taxonómicas en el futuro. El total de endemismos tanto a nivel específico como genérico presentó cambios importantes. Los géneros Thoreauea (Apocynoides), Suberogerens y Trichosacme Brandegee (Asclepiadoideae) se reportan como endémicos (Tabla 3). En cambio, Thenardia pierde su estatus como 
género endémico de México (Juárez-Jaimes et al. 2007) con el descubrimiento de T. chiapensis J.K. Williams en Honduras (Morales 2009). Asimismo, Microdactylon Brandegee, género monotípico y restringido a Oaxaca, perdió su estatus de endémico al ser sinonimizado a Polystemma (Endress et al. 2018). Esta propuesta es apoyada aquí, ya que la morfología floral corresponde a Polystemma. A nivel específico, el endemismo se incrementó con respecto a lo planteado por Juárez-Jaimes et al. (2007). De las 204 especies endémicas, 67 (32\%) son microendémicas (Tabla 3,4 ) y muchas de ellas se conocen solo del ejemplar tipo. De este grupo restringido de especies, los estados de las vertientes del Pacífico y del Golfo presentan la mayor diversidad (e.g., Chiapas, Oaxaca, Jalisco, Veracruz). Tanto para las especies endémicas como para la riqueza total de la familia, los factores físicos y biológicos del país juegan un papel importante en la diversidad y distribución del grupo. Estos factores podrían estar generando un mosaico de condiciones climáticas y de interacciones que proporcionan hábitats apropiados y muy restringidos para la supervivencia de las especies, así como laboratorios de novedades taxonómicas (Cunas y Museos sensu Sosa et al. 2018). Situación similar observada en grupos como Asteraceae (Villaseñor 2018), Lamiaceae (Martínez-Gordillo et al. 2017) y Loganiaceae (IslasHernández et al. 2017).

Es necesaria la planeación de colectas dirigidas a numerosas áreas de ambas vertientes del país, así como en las serranías del sureste y del norte donde la diversidad y endemismos es alta. La aplicación de modelos de distribución potencial podría ayudar a esta tarea. Aproximaciones con esta herramienta en Cascabela (Alvarado-Cárdenas et al. 2017) y Tabernaemontana (Alvarado-Cárdenas et al. 2019) sugieren que ambas vertientes tienen condiciones idóneas para la presencia de sus especies, y potencialmente indicar la existencia de taxones nuevos (Raxworthy et al. 2007), tanto de estos como de otros géneros.

Conservación. El estado de conservación de Apocynaceae en México es poco conocido y solo se han asignado a una de las categorías de riesgo de la IUCN el $36 \%$ del total de especies y solo dos se encuentran incluidas en la NOM-059 (SEMARNAT 2010a, $\underline{\text { b) }}$. Se sabe que muchas especies solo se conocen del ejemplar tipo, y de las 204 especies endémicas, el $50 \%$ tiene distribución restringida a 1 o 2 estados y solo al $30 \%$ del total se le ha otorgado un estado de conservación (Tabla 4, $\underline{5}$, Material suplementario 2) muchas de ellas en zonas de alta perturbación antrópica como Veracruz. Además, la versión más reciente de la NOM-059 (SEMARNAT 2010b) no incluye más miembros de Apocynaceae en su listado. Es necesaria la integración del estado de riesgo de las especies de este grupo en los documentos oficiales del país para una potencial protección. Nuevamente, la exploración de las serranías y remanentes de la vegetación natural permitirá una mejor comprensión de qué potenciales riesgos podrían enfrentar los taxones. Asimismo, la aplicación de sistemas de información geográfica y modelos de distribución potencial podrán aportar más argumentos para la selección de la categoría de riesgo, como ya se ha aplicado para Apocynaceae (Alvarado-Cárdenas et al. 2019) y otros grupos (Solano \& Feria 2006, Maycock et al. 2012, Anguiano-Constante et al. $\underline{2018)}$.

Otro de los aspectos que requieren atención es el conocimiento de las interacciones con otros organismos. El último análisis del conocimiento de la polinización de este grupo a nivel mundial arrojó que no más de 20 especies de apocináceas mexicanas contaban con esta información (Liede 1994, Kunze 1999, Ollerton et al. 2018). Esto refleja la enorme brecha de desconocimiento que tenemos al respecto. El análisis de estas interacciones en conjunto con la información morfológica y filogenética permitirá un mejor entendimiento de los procesos involucrados en la evolución de la familia y la historia natural de las especies.

La evaluación filogenética, morfológica y ecológica que se realiza en los diferentes niveles taxonómicos de la familia, aportará evidencia para resolver numerosos problemas de circunscripción, como complejos de especies, relaciones filogenéticas entre y dentro de los géneros, y grupos más inclusivos. Los descubrimientos continuos de especies aportarán información sobre la variación morfológica de los diferentes géneros, así como aspectos sobre su biogeografía. La integración de sistemáticos permitirá abordar con perspectivas novedosas aspectos filogenéticos, morfológicos, biogeográficos, fitoquímicos, de interacciones, entre otros. Además de todo el trabajo sistemático por hacer, es un momento complicado para la biodiversidad (Thomas et al. 2004, Pimm et al. 2014, Villaseñor 2015), pero es también un momento importante para los descubrimientos y esfuerzos por aportar conocimiento que favorezcan la mitigación de la degradación de nuestros ecosistemas. Además de la aplicación de herramientas analíticas nuevas, el papel de las colecciones biológicas, listados florísticos y tratamientos taxonómicos actualizados son fundamentales para aportar argumentos en la toma de decisiones para el uso sustentable, investigación y conservación de la biodiversidad.

\section{Agradecimientos}

Se agradece a Jaime Jiménez R, Ixchel S. González R, Matus H. Barón y César González M. por sus comentarios en la mejora del manuscrito. Se agradece a dos revisores anónimos por sus observaciones que mejoraron de forma sustancial el trabajo. También a todo el personal de los 
herbarios consultados y a María Eugenia Muñiz Díaz de León su apoyo en el trabajo realizado en el Taller de Plantas I y II. Agradecemos también a Aldi De Oyarzabal Salcedo por las bellas acuarelas, a Allen Coombes, Leccinum García, Francisco Morales, y Víctor Steinmman por las fotografías proporcionadas.

\section{Material Suplementario}

El material suplementario de este artículo puede consultarlo aquí: https://doi.org/10.17129/botsci.2525

\section{Literatura citada}

Alvarado-Cárdenas LO. 2004. Apocynaceae. Flora del Valle de Tehuacán-Cuicatlán. 38: 1-57.

Alvarado-Cárdenas LO. 2007. Stemmadenia oaxacana Alvarado-Cárdenas (Apocynaceae) una nueva especie para el Estado de Oaxaca, México. Candollea 62: 189-192.

Alvarado-Cárdenas LO. 2016. Odontadenia macrantha (Apocynaceae; Apocynoideae): distribución y nuevos registros en México. Acta Botanica Mexicana 117: 93-99. DOI: https://doi.org/10.21829/abm117.2016.1170

Alvarado-Cárdenas LO, Juárez-Jaimes V. 2011. Lista de especies de la familia Apocynaceae (subfamilias Rauvolfioideae y Apocynoideae). In: García-Mendoza A, Meave JA, eds. Diversidad Florística de Oaxaca: de Musgos a Angiospermas (Colecciones y Lista de especies). México: Instituto de Biología, CIIDIROaxaca, Jardín Etnobotánico de Oaxaca, SERBO AC, pp. 203-219. ISBN: 9876070224348

Alvarado-Cárdenas LO, Juárez-Jaimes V. 2012. Una especie nueva de Tabernaemontana L. (Apocynaceae; Rauvolfioideae) para México, probablemente extinta en su hábitat. Revista Mexicana de Biodiversidad 83: 334-340. DOI: http://dx.doi.org/10.22201/ib.2007870 6e.2012.2.978

Alvarado-Cárdenas LO, Lozada-Pérez L, Cadena J, IslasHernández S, Martínez-González CR, Cortez EB, González-Martínez CA, González-Ramírez IS. 2019. The triad of knowledge: Systematic, diversity and conservation status of the Mexican species of Tabernaemontana (Apocynaceae; Rauvolfioideae: tribe Tabernaemontaneae). Phytotaxa, 388: 1-46. DOI: https:// doi.org/10.11646/phytotaxa.388.1.1

Alvarado-Cárdenas LO, Morales F. 2014. El género Mandevilla (Apocynaceae: Apocynoideae, Mesechiteae) en México. Botanical Sciences 92: 59-79. DOI: $\underline{\text { https:// }}$ doi.org/10.17129/botsci.28

Alvarado-Cárdenas LO, Ochoterena H. 2007. A Phylogenetic Analysis of the Cascabela-Thevetia Species Complex (Apocynaceae; Plumerieae) Based on
Morphology. Annals of the Missouri Botanical Garden 94: 298-322. DOI: https://doi.org/10.3417/0026-6493 (2007)94[298:APAOTC]2.0.CO;2

Alvarado-Cárdenas LO, Villaseñor JL, López-Mata L, Cadena J, Ortiz E. 2017. Systematics, distribution and conservation of Cascabela (Apocynaceae: Rauvolfioideae: Plumerieae) in Mexico. Plant Systematics and Evolution 303: 337-369. DOI: https:// doi.org/10.1007/s00606-016-1375-6

Anguiano-Constante MA, Munguía-Lino G, Ortiz E, Villaseñor JL, Rodríguez A. 2018. Riqueza, distribución geográfica y conservación de Lycianthes serie Meizonodontae (Capsiceae, Solanaceae). Revista Mexicana de Biodiversidad 89: 516-529. DOI: https:// doi.org/10.22201/ib.20078706e.2018.2.2340

APG IV [The Angiosperm Phylogeny Group], Chase MW, Christenhusz MJM, Fay MF, Byng JW, Judd WS, Soltis DE, Mabberley DJ, Sennikov AN, Stevens PF. 2016. An update of the Angiosperm Phylogeny Group classification for the orders and families of flowering plants. Botanical Journal of the Linnean Society 181: 1-20. DOI: http://doi.org/10.1111/boj.12385

Bentham G. 1845. Botany of the voyage of H.M.S Sulphur. Smith, Elder and Co., London. DOI: https://doi.org/ $\underline{10.5962 / \text { bhl.title. } 908}$

Bebawi FF, Campbell SD, Mayer RJ. 2017. Seed fall, seed predation, twigging and litter fall of Cascabela thevetia (L.) Lippold. The Rangeland Journal 38: 569-577. DOI: https://doi.org/10.1071/RJ16021

Biblioteca Digital Mundial. 2016. Historia general de las cosas de Nueva España por el fray Bernardino de Sahagún: el Códice Florentino. https://www.wdl.org/es/ item/10096/ (accessed May, 2019).

Biodiversity Heritage Library. 2019. https://www.biodiver sitylibrary.org/ (accessed January 2019).

BFG [The Brazil Flora Group]. 2015. Growing knowledge: an overview of Seed Plant diversity in Brazil. Rodriguesia 66: 1085-1113. DOI: http://doi.org/10.15 90/2175-7860201566411

Brandegee TS. 1906. New species of Mexican plants collected by Dr. C. Purpus. Zoe 5: 231-241.

Brandegee TS. 1908. New species of Mexican plants. Zoe 5: 244-262.

Brandegee TS. 1909. Plantae Mexicanae Purpusianae I-XII, University of California Publications in Botany 3: 377-396. ISBN-10: 113054236X; ISBN-13: 978-1130542363

Bruyns PV. 2005. Stapeliads of southern Africa and Madagascar (Vol. 1). Umdaus Press. ISBN: 978-191-9766-37-9

Cervantes-Meza CO. 2018. Sinopsis del género Asclepias (Asclepiadoideae, Apocynaceae) de Hidalgo, México. 
BSc Thesis. Universidad Autónoma del Estado de Hidalgo.

Coates-Estrada R, Estrada A, Meritt D. 1993. Foraging by parrots (Amazona autumnalis) on fruits of Stemmadenia donnell-smithii (Apocynaceae) in the tropical rain forest of Los Tuxtlas, Mexico. Journal of Tropical Ecology 9: 121-124. DOI: https://doi.org/10.1017/S02664674000 $\underline{07070}$

Coombs G, Peter CI. 2010. The invasive 'mothcatcher' (Araujia sericifera Brot.; Asclepiadoideae) co-opts native honeybees as its primary pollinator in South Africa. AoB plants 2010: plq021. DOI: https://doi.org/ $\underline{10.1093 / \mathrm{aobpla} / \mathrm{plq} 021}$

Cortez-Castro EB. 2018. La familia Apocynaceae en el estado de Hidalgo, México. BSc Thesis. Universidad Nacional Autónoma de México.

Cortez EB, Lozada-Pérez L, Alvarado-Cárdenas LO. 2018. Two New Species of Matelea (Asclepiadoideae: Gonolobeae; Gonolobinae) from Mexico. Systematic Botany 43: 818-825. DOI: https://doi.org/10.1600/ 036364418X697544

Dávila-Aranda P, Villaseñor JL, Medina-Lemos R, Ramírez-Roa A, Salinas-Tovar A, Sánchez-Ken J, Tenorio-Lezama P. 1993. X. Flora del Valle de Tehuacán-Cuicatlán.: In: Instituto de Biología, Universidad Nacional Autónoma de México. Listados Florísticos de México. ISBN de la colección: 968-36-1004-8

De Candolle AP. 1844. Prodromus Systematis Regni Vegetabilis. v8. París: Fortin Masson. DOI: https:// doi.org/10.5962/bhl.title.286

De la Cruz M. 1552. Libellus de medicinalibus indorum herbis: Manuscrito Azteca de 1552 (según traducción latina de Juan Badiano), versión española con estudios y comentarios por diversos autores (A facsímile of the manuscript in the Biblioteca Apostolica Vaticana). México: Instituto Mexicano del Seguro Social 1964.

Decaisne J. 1838. Études sur quelques genres et espèces de la famille des Asclépiadées. Annales des Sciences Naturelles; Botanique, série 2. 9: 321-348.

Decaisne J. 1844. Asclepiadaceae. In: De Candolle AP. Prodromus Systematis Naturalis Regni Vegetabilis. v8. Paris: Fortin Masson, pp. 599-665. DOI: https://doi.org/ $\underline{10.5962 / \text { bhl.title. } 286}$

Diego-Pérez N. 2004. Apocynaceae. Flora de Guerrero. 20: 1-117. ISBN: 970-32-2289-7

Do Espírito Santo FDS, Rapini A, Ribeiro PL, LiedeSchumann S, Goyder DJ, Fontella-Pereira J. 2019. Phylogeny of the tribe Marsdenieae (Apocynaceae), reinstatement of Ruehssia and the taxonomic treatment of the genus in Brazil. Kew Bulletin 74: 30. DOI: https:// doi.org/10.1007/s12225-019-9807-4
Domínguez XA, Rojas P, Collins V, Ma Del Morales R. 1958. A phytochemical study of eight Mexican plants. Economic Botany 14: 157-159. https://doi.org/10.1007/ $\underline{\mathrm{BF} 02860018}$

Endress ME, Bruyns PV. 2000. A revised classification in the Apocynaceae s.1. The Botanical Review 66: 1-56. DOI: http://doi.org/10.1007/BF02857781

Endress ME, Hansen BF 2007. Pinochia, a new genus of Apocynaceae, Apocynoideae from the Greater Antilles, Mexico and Central America. Edinburgh Journal of Botany, 64: 269-274. DOI: https://doi.org/10.1017/ S0960428607000959

Endress ME, Sennblad B, Nilsson S, Civeyrel L, Chase MW, Huysmans S, Grafstrom E, Bremer B. 1996. A phylogenetic analysis of Apocynaceae s. str. and some related taxa in Gentianales: a multidisciplinary approach. In: Conference Proceedings: $2^{\text {nd }}$ International Rubiaceae Conference. Belgica: National Botanical Garden Belgium. 7: pp. 59-102. ISBN: 90-72619-29-3

Endress ME, Liede-Schumann S, Meve U. 2014. An updated classification for Apocynaceae. Phytotaxa 159: 175-194. DOI: http://doi.org/10.11646/phytotaxa.159.3.2

Endress ME, Meve U, Middleton DJ, Liede-Schumann S. 2018. Apocynaceae. In: Kadereit JW, Bittrich V, eds. Flowering Plants. Eudicots, The Families and Genera of Vascular Plants 15. Springer International Publishing AG, pp. 207-411. DOI: https://doi.org/10.1007/ 978-3-319-93605-5 33; Print ISBN 978-3-319-93604-8

Fernández-Brewer AM, Juárez-Jaimes V, Cortés-Zárraga L. 2008. Usos de las especies del género Asclepias L. (Apocynaceae, Asclepiadoideae), información del Herbario Nacional de México, MEXU. Polibotánica 25: 155-171.

Fishbein M. 2001. Evolutionary innovation and diversification in the flowers of Asclepiadaceae. Annals of the Missouri Botanical Garden 88: 603-623. DOI: https://doi.org/10.2307/3298636

Fishbein M. 2008. A new, diminutive, Mexican milkweed (Asclepias, Apocynaceae s.l.). Novon. 18: 43-47. DOI: https://doi.org/10.3417/2006089

Fishbein M. 2017. Taxonomic adjustments in North American Apocynaceae. Phytologia 99: 86-88.

Fishbein M, Stevens WD. 2005. Resurrection of Seutera Reichenbach (Apocynaceae, Asclepiadoideae). Novon 15: 531-533.

Fishbein M, Chuba D, Ellison C, Mason-Gamer RJ, Lynch SP. 2011. Phylogenetic relationships of Asclepias (Apocynaceae) inferred from non-coding chloroplast DNA sequences. Systematic Botany 36: 1008-1023. DOI: https://doi.org/10.1600/036364411X605010

Fishbein M, Livshultz T, Straub SC, Simões AO, Boutte J, McDonnell A, Foote A. 2018. Evolution on the backbone: Apocynaceae phylogenomics and new 
perspectives on growth forms, flowers, and fruits. American Journal of Botany 105: 495-513. DOI: https:// doi.org/10.1002/ajb2.1067

Fonseca G, Rodríguez-Hahn L, Tablero M, Rodríguez A, Arreguín B. 1991. Labriformin, a cardiac glucoside from Asclepias glaucescens. Journal of Natural Products 54: 860-862. DOI: https://doi.org/10.1021/np50075a018

Gaillard Y, Krishnamoorthy A, Bevalot F. 2004. Cerbera odollam: a 'suicide tree' and cause of death in the state of Kerala, India. Journal of Ethnopharmacology 95: 123-126. DOI: https://doi.org/10.1016/j.jep.2004.08.004

García-Alvarado JS, Verde-Star MJ, Heredia NL. 2001. Traditional uses and scientific knowledge of medicinal plants from Mexico and Central America. Journal of Herbs, Spices and Medicinal Plants 8: 37-89. DOI: https://doi.org/10.1300/J044v08n02 02

Global Plants JSTOR. 2019. http://plants.jstor.org (accessed Jan 2019).

Gómez-Pompa A. 2016. Mi vida en las selvas tropicales. Memorias de un botánico. México. http://www. reservaeleden.org/agp/pdf/AutobiografiaAGP.pdf

Gómez-Pompa A, Nevling LI. 1970. Flora de Veracruz. Anales del Instituto de Biología, UNAM. Serie: Botánica, 41: 1-2.

González-Martínez CA. 2019. Dictyanthus Decne. (Apocynaceae: Asclepiadoideae: Gonolobinae) género casi endémico de México e ignorado en las filogenias. MSc. Thesis. Universidad Nacional Autónoma de México.

González-Martínez C, Lozada-Pérez L, Alvarado-Cárdenas L. 2019. Dictyanthus stevensii (Apocynaceae; Asclepiadoideae; Gonolobinae), a new species from the Zoque forest, Chimalapas region in the state of Oaxaca, Mexico. Phytotaxa 394: 77-88. DOI: http://dx.doi.org/ 10.11646/phytotaxa.394.1.5

González-Rocha E, Cerros-Tlatilpa R. 2015. La familia Apocynaceae (Apocynoideae y Rauvolfioideae) en el estado de Morelos, México. Acta Botanica Mexicana 110: 21-70. DOI: https://doi.org/10.21829/abm110.2015. 191

Grice AC. 1997. Post-fire regrowth and survival of the invasive tropical shrubs Cryptostegia grandiflora and Ziziphus mauritiana. Australian Journal of Ecology 22: 49-55. DOI: https://doi.org/10.1111/j.1442-9993.1997. tb00640.x

Hansen BF, Wunderlin RP. 1986. Pentalinon Voigt, an earlier name for Urechites Müll. Arg. (Apocynaceae). Taxon 35: 166-168. DOI: https://doi.org/10.2307/ $\underline{1221059}$

HUH [Harvard University Herbaria \& Libraries]. 2019. http://www.huh.harvard.edu (accessed Feb 2019).

Heinrich M, Rimpler H, Barrera NA. 1992. Indigenous phytotherapy of gastrointestinal disorders in a lowland
Mixe community (Oaxaca, Mexico): Ethnopharmacologic evaluation. Journal of Ethnopharmacology 36: 63-80. DOI: https://doi.org/ 10.1016/0378-8741(92)90062-V

Herbario CICY. 2010-2020. Flora de la Península de Yucatán. http://www.cicy.mx/sitios/flora\%20digital/

Hernández F. 1517-1587. Historia de las Plantas de Nueva España. Facsímil Tomo 1 (Libro 1 y 2 ) México: Imprenta Universitaria 1943.

Hernández-Barón GM. 2017. La subfamilia Asclepiadoideae (Apocynaceae) de la región de Los Tuxtlas, Veracruz, México, un acercamiento taxonómico. BSc Thesis. Universidad Autónoma del Estado de Morelos.

Hernández-Barón GM, Juárez-Jaimes V, CamposVillanueva A. 2019. La subfamilia Asclepiadoidea (Apocynaceae) de la región de Los Tuxtlas, Veracruz, México. Revista Mexicana de Biodiversidad 90: e902897. DOI: http://dx.doi.org/10.22201/ib.20078706e. $\underline{2019.90 .2897}$

Hooker WJ, Arnott GAW. 1841. The Botany of Captain Beechey's Voyage. London: Henry G. Bohn.

Ibarra-Manríquez G, Rendón-Sandoval FJ, Cornejo-Tenorio G, Carrillo-Reyes P. 2015. Lianas de México. Botanical Sciences 93: 365-417. DOI: https://doi.org/10.17129/ botsci. 123

In Defense of Plants. 2019. http://www.indefenseof plants.com/ (accessed May, 2019).

International Plant Names Index. 2019. https://www. ipni.org/ (accessed Jan 2019).

Islas-Hernández CS, García RB, Alvarado-Cárdenas LO. 2017. New additions of Spigelia (Loganiaceae) in Mexico. Phytotaxa 331: 243-252. DOI: https://doi.org/ 10.11646/phytotaxa.331.2.8

Jacquin NJ. 1760. Enumeratio Systematica Plantarum, quas in insulis Caribaeis. Switzerland: Inter Documentation Company AG41 pp. DOI: https://doi.org/10.5962/bhl. $\underline{\text { title. } 123272}$

Jacquin NJ. 1806. Stapeliarum in Hortis Vindobonensibus Cultarum. Vienna. 64 lam. Vindobonae: Prostant apud Wappler et Beck.

Janick J, Tucker AO. 2017. Were Juan Gerson the illustrator and Gasper de Torres the author of the Voynich Codex?. Notulae Botanicae Horti Agrobotanici Cluj-Napoca 45: 343-352. COI: https://doi.org/10.15835/nbha45210693

Juárez-Jaimes V. 1998. Revisión taxonómica del género Marsdenia R.Br. subsección Macrophyllae Rothe (Asclepiadaceae Marsdenieae). MSc Thesis. Universidad Nacional Autónoma de México.

Juárez-Jaimes V, Ángeles-Trujillo ALM. 2013. El género Marsdenia (Apocynaceae: Asclepiadoideae) en Guerrero. Revista Mexicana de Biodiversidad 84: 425-438. DOI: http://doi.org/10.7550/rmb.30946 
Juárez-Jaimes V, Alvarado-Cárdenas LO. 2010. Dos especies nuevas de Marsdenia (Apocynaceae, Asclepiadoideae: Marsdeniae) de México. Candollea 65: 63-68. DOI: https://doi.org/10.15553/c2010v651a6

Juárez-Jaimes V, Alvarado-Cárdenas LO, Villaseñor JL. 2007. La familia Apocynaceae sensu lato en México: diversidad y distribución. Revista Mexicana de Biodiversidad 78: 459-482. DOI: http://dx.doi.org/ 10.22201/ib.20078706e.2007.002.402

Juárez-Jaimes BV, Lozada-Pérez L. 2003. Asclepiadaceae. Flora del Valle de Tehuacán-Cuicatlán. 37: 1-57. ISBN: 970-32-1293-X

Juárez-Jaimes V, Lozada-Pérez L. 2015. Marsdenia microcarpa, a new species of Marsdenia (Apocynaceae, Asclepiadoideae) from montane Guerrero, Mexico. Phytotaxa 217: 92-95. DOI: https://doi.org/10.11646/ phytotaxa.217.1.9

Kew [Royal Botanical Gardens]. 2019. Neotropical Flowering Plants. http://www.kew.org/science/tropame rica/neotropikey.htm (accessed Feb 2019).

Krengel F, Herrera Santoyo J, Olivera Flores TDJ, Chávez Ávila VM, Pérez Flores FJ, Reyes Chilpa R. 2016. Quantification of Anti-Addictive Alkaloids Ibogaine and Voacangine in In Vivo-and In Vitro-Grown Plants of Two Mexican Tabernaemontana Species. Chemistry \& Biodiversity 13: 1730-1737. DOI: https://doi.org/10. 1002/cbdv.201600146

Krengel F, Chevalier Q, Dickinson J, Santoyo JH, ReyesChilpa R. 2019. Metabolite Profiling of Antiaddictive Alkaloids from Four Mexican Tabernaemontana Species and the Entheogenic African Shrub Tabernanthe iboga (Apocynaceae). Chemistry \& Biodiversity 16: e1800506. DOI: https://doi.org/10.1002/cbdv.201800506

Krings A. 2011. Matelea s. 1. (Apocynaceae, Asclepiadoideae) in the West Indies. Systematic Botany 36: 730-756. DOI: https://doi.org/10.1600/036364411 $\underline{\mathrm{X} 583691}$

Krings A, Morillo G. 2015. A new species in the Matelea palustris complex (Apocynaceae, Asclepiadeae) and a synopsis of the complex in the Guianas and northern Brazil. Systematic Botany 40: 214-219. DOI: https:// doi.org/10.1600/036364415X686521

Krings A, Thomas DT, Xiang Q. 2008. On the generic circumscription of Gonolobus (Apocynaceae, Asclepiadoideae): Evidence from molecules and morphology. Systematic Botany 33: 403-415. DOI: https://doi.org/10.1600/036364408784571527

Kunze H. 1999. Pollination ecology in two species of Gonolobus (Asclepiadaceae). Flora 194: 309-316. DOI: https://doi.org/10.1016/S0367-2530(17)30919-2

Kunze H. 2005. Morphology and evolution of the corolla and corona in the Apocynaceae s.l. Botanische
Jahrbücher 126: 347-383. DOI: https://doi.org/10.1127/ 0006-8152/2005/0126-0347

Liede S. 1994. Some observations on pollination in Mexican Asclepiadaceae. Madroño 41: 266-276.

Liede S. 1997. American Cynanchum (Asclepiadaceae): A preliminary infrageneric classification. Novon 7: 172-181. DOI: https://doi.org/10.2307/3392191

Liede-Schumann S, Meve U. 2004. Revision of Metastelma (Apocynaceae-Asclepiadoideae) in southwestern North America and Central America. Annals of the Missouri Botanical Garden 91: 31-86.

Liede-Schumann S, Meve U. 2008. Nomenclatural novelties and one new species in Orthosia (Apocynaceae, Asclepiadoideae). Novon 18: 202-210. DOI: https://doi.org/10.3417/2006150

Liede-Schumann S, Meve U. 2013. The Orthosiinae revisited (Apocynaceae, Asclepiadoideae, Asclepiadeae). Annals of the Missouri Botanical Garden 99: 44-81. DOI: https://doi.org/10.3417/2010130

Liede-Schumann S, Rapini A, Goyder DJ, Chase MW. 2005. Phylogenetics of the New World subtribes of Asclepiadeae (Apocynaceae-Asclepiadoideae): Metastelmatinae, Oxypetalinae, and Gonolobinae. Systematic Botany 30: 184-195. DOI: https://doi.org/ 10.1600/0363644053661832

Liede S, Täuber A. 2002. Circumscription of the genus Cynanchum (Apocynaceae-Asclepiadoideae). Systematic Botany 27: 789-800. DOI: https://doi.org/10.1043/ 0363-6445-27.4.789

Linnaeus C. 1753. Species Plantarum. Tomo I. Imprensis Laurentii Salvii, Holmiae. https://doi.org/10.5962/ bhl.title. 669

Livshultz T, Middleton D.J, Endress ME, Williams JK. 2007. Phylogeny of Apocynoideae and the APSA clade (Apocynaceae s.1.). Annals of the Missouri Botanical Garden 94: 324-359. DOI: https://doi.org/10.3417/ 0026-6493(2007)94[324:POAATA]2.0.CO;2

Llanos-Romero RE, Cárdenas R, Zúñiga B, HerreraSantoyo J, Guevara-Fefer P. 2014. Acetylcholinesterase inhibitory activity of Haplophyton cimicidum. Natural Product Research 28: 757-759. DOI: http://dx.doi.org/ 10.1080/14786419.2013.879131

Lozada-Pérez L. 2003. Sistemática de Pherotrichis Decne. (Apocynaceae, Asclepiadoideae). MSc. Thesis. Universidad Nacional Autónoma de México.

Lozada-Pérez L. 2010. Polystemma mirandae (Apocynaceae, Asclepiadoideae) una nueva especie de México. Novon 20: 429-431. DOI: https://doi.org/ $\underline{10.3417 / 2009004}$

Lozada-Pérez L, Alvarado-Cárdenas LO. 2015. Reevaluation of the Mandevilla subsessilis species complex and resurrection of M. platydactyla (Apocynaceae: 
Apocynoideae). Phytotaxa 239: 155-164. DOI: https:// doi.org/10.11646/phytotaxa.239.2.4

Lozada-Pérez L, Alvarado-Cárdenas LO. 2016. A new species of Matelea s.l (Apocynaceae, Asclepiadoideae) from Mexico. Phytotaxa 288: 168-174. DOI: https:// doi.org/10.11646/phytotaxa.288.2.7

Lozada-Pérez L, Diego-Pérez N. 2012. Mandevilla guerrerensis (Apocynaceae, Apocynoideae), una nueva especie de México. Darwiniana 50: 318-322. DOI: https://doi.org/10.14522/darwiniana.2014.502.494

Madrinán S. 2009. Jacquin al descubierto: transcripción de la Biographia. Revista de Estudios Sociales 32: 214-239.

Marroquín JS, Calderón de Rzedowski G. 2001. Apocynaceae. In: Calderón de Rzedowski G, Rzedowski J, eds. Flora Fanerogámica del Valle de México. Pátzcuaro. Michoacán: Instituto de Ecología AC. Comisión Nacional para el Conocimiento y Uso de la Biodiversidad, pp. 558-563. ISBN 970-9000-17-9.

Martínez-Gordillo M, Bedolla-García B, Cornejo-Tenorio G, Fragoso-Martínez I, García-Peña MDR, GonzálezGallegos JG, Lara-Cabrera SI \& Zamudio S. 2017. Lamiaceae de México. Botanical Sciences 95: 780-806. DOI: https://doi.org/10.17129/botsci.1871

Mauz K. 2011. Cyrus Pringle's vascular plant types from western United States and Mexico, 1881-1884. Harvard Papers in Botany 16: 71-142. DOI: https://doi.org/ 10.3100/025.016.0111

Maycock CR, Kettle CJ, Khoo E, Pereira JT, Sugau JB, Nilus R, Ong R, Amaludin N, Newman M, Burslem DF. 2012. A revised conservation assessment of Dipterocarps in Sabah. Biotropica 44: 649-657. DOI: https://doi.org/ 10.1111/j.1744-7429.2011.00852.x

McDonnell A, Fishbein M. 2016. Polystemma canisferum (Apocynaceae, Asclepiadoideae): a distinctive new gonoloboid milkweed vine from Sonora, Mexico. Phytotaxa 246: 78-84. DOI: http://dx.doi.org/10.11646/ phytotaxa.246.1.6

McDonnell A, Fishbein M, Quinn M, Hare T, Keith K. 2015. Matelea chihuahuensis (Apocynaceae): An addition to the flora of the United States and a synopsis of the species. Journal of the Botanical Research Institute of Texas 9: 187-194.

McDonnell A, Parks M, Fishbein M. 2018. Multilocus phylogenetics of New World milkweed vines (Apocynaceae, Asclepiadoideae, Gonolobinae). Systematic Botany 43: 77-96. DOI: https://doi.org/ $\underline{10.1600 / 036364418 \times 697021}$

McVaugh R. 1969. El itinerario y las colectas de Sessé y Mociño en México. Boletín de la Sociedad Botánica de México 30: 137-142. DOI: https://doi.org/10.17129/ $\underline{\text { botsci. } 1104}$
McVaugh R. 1980. Karwinski's itineraries in Mexico, 1827-1832 and 1841-1843. Contributions from the University of Michigan Herbarium 14: 141-152.

Menge EO, Bellairs SM, Lawes MJ. 2017. Disturbancedependent invasion of the woody weed, Calotropis procera, in Australian rangelands. The Rangeland Journal 39: 201-211. DOI: https://doi.org/10.1071/ $\underline{\mathrm{RJ} 16120}$

Miranda F, Valdés J. 1964. "Comentarios botánicos" In: de la Cruz M. Libellus de Medicinalibus Indorum.Códices mexicanos. México: Fondo de Cultura Económica, Instituto Mexicano del Seguro Social, pp. 243-284. ISBN: 978-968-1636-07-4

Morales JF. 1996. Novelties in Prestonia (Apocynaceae). Novon 6: 285-287. DOI: https://doi.org/10.2307/3392095

Morales JF. 1997a. A reevaluation of Echites and Prestonia sect. Coalitae (Apocynaceae). Brittonia 49: 328-336. DOI: https://doi.org/10.2307/2807832

Morales JF. 1997b. A synopsis of the genus Prestonia (Apocynaceae) section Tomentosae in Mesoamerica. Novon 7: 59-66. DOI: https://doi.org/10.2307/3392074

Morales JF. 2002a. Studies in Neotropical Apocynaceae I: a revision of the genus Laubertia. Rhodora 104: 170-185.

Morales JF. 2002b. Studies in Neotropical Apocynaceae II: a review of the genus Fernaldia. Rhodora 104: 186-200.

Morales JF. 2005. Estudios en las Apocynaceae Neotropicales XV: Sinopsis del género Thoreauea (Apocynoideae, Echiteae), con una nueva especie de Veracruz, México. Brittonia 57: 258-263. DOI: https:// doi.org/10.1663/0007-196X(2005)057[0258:EELANX] 2.0.CO;2

Morales JF. 2009. Estudios en las Apocynaceae neotropicales XXXIX: revisión de las Apocynoideae y Rauvolfioideae de Honduras. Anales del Jardín Botánico de Madrid. 66: 217-262. DOI: https://doi.org/10.3989/ ajbm.2205

Morales JF, Méndez M. 2005. Estudios en las Apocynaceae Neotropicales XXII: nuevos realineamientos taxonómicos en el género Stemmadenia (Apocynaceae, Rauvolfioideae, Tabernaemontaneae). Candollea 60: 345-371.

Morales JF, Williams JK. 2004. Allotoonia, a new neotropical genus of Apocynaceae based on a subgeneric segregate of Echites. SIDA, Contributions to Botany 21: 133-158.

Morales JF, Zamora NA. 2017. A synopsis of Aspidosperma (Apocynaceae) in Mexico and Central America with a taxonomic clarification of Aspidosperma cruentum and a new cryptic species. Phytoneuron 68: 1-13.

Morales JF, Endress ME, Liede-Schumann S. 2017. Sex, drugs and pupusas: Disentangling relationships in 
Echiteae (Apocynaceae). Taxon 66: 623-644. DOI: https://doi.org/10.12705/663.7

Morillo G. 1976. A revision of Blepharodon (Asclepiadaceae). M.Sc. Thesis Louis University.

Morillo G. 1997. Revisión preliminar de Metalepis Griseb. (Asclepiadaceae). Pittieria 26: 65-99.

Morillo G. 2012. Aportes al conocimiento de las Gonolobinae (Apocynaceae - Asclepiadoideae). Pittieria 36: 1357.

Morillo G. 2015. Aportes al conocimiento de las Gonolobinae Parte III (Apocynaceae, Asclepiadoideae). Pittieria 39: 191-258.

Mroue MA, Ghuman MA, Alam M. 1993. Crooksidine, an indole alkaloid from Haplophyton crooksii. Phytochemistry 33: 217-219. DOI: https://doi.org/ 10.1016/0031-9422(93)85426-R

Murphy H. 1986. A revision of the genus Fischeria (Asclepiadaceae). Systematic Botany 11: 229-241. DOI: https://doi.org/10.2307/2418961

NaturaLista. 2019. https://www.naturalista.mx (accessed April, 2019).

Navarro-García VN, González A, Fuentes M, Avilés M, Ríos MY, Zepeda G, Rojas MG. 2003. Antifungal activities of nine traditional Mexican medicinal plants. Journal of Ethnopharmacology 87: 85-88. DOI: https:// doi.org/10.1016/S0378-8741(03)00114-4

Ollerton J, Liede-Schumann S, Endress ME, Meve U, Rech AR, Shuttleworth A, Keller HA, Fishbein M, AlvaradoCárdenas LO, Amorim FW, Bernhardt P, Celep F, Chirango Y, Chiriboga-Arroyo F, Civeyrel L, Cocucci A, Cranmer L, da Silva-Batista IC, de Jager L, Deprá MS, Domingos-Melo A, Dvorsky C, Agostini K, Freitas L, Gaglianone MC, Galetto L, Gilbert M, GonzálezRamírez I, Gorostiague P, Goyder D, Hachuy-Filho L, Heiduk A, Howard A, Ionta G, Islas-Hernández SC, Johnson SD, Joubert L, Kaiser-Bunbury CN, Kephart S, Kidyoo A, Koptur S, Koschnitzke C, Lamborn E, Livshultz T, Machado IC, Marino S, Mema L, Mochizuki K, Morellato LPC, Mrisha CK, Muiruri EW, Nakahama N, Nascimento VT, Nuttman C, Oliveira PE, Peter CI, Punekar S, Rafferty N, Rapini A, Ren ZX, Rodríguez-Flores CI, Rosero L, Sakai S, Sazima M, Steenhuisen SL, Tan CW, Torres C, Trøjelsgaard K, Ushimaru A, Vieira MF, Wiemer AP, Yamashiro T, Nadia T, Queiroz J, Quirino Z. 2018. The diversity and evolution of pollination systems in large plant clades: Apocynaceae as a case study. Annals of Botany 123: 311-325. DOI: https://doi.org/10.1093/aob/mcy127

Pichon M. 1948a. Classification des Apocynacées: V, Cerbéroidées. Notulae Systematicae. Herbier du Museum de Paris 13: 212-229
Pichon M. 1948b. Classification des Apocynacées: VI, Genre Tabernaemontana. Notulae Systematicae. Herbier du Museum de Paris 13: 230-254.

Pichon M. 1948c. Classification des Apocynacées: IX, Rauvolfiées, Alstoniées, Allamandées et Tabernémontanoïdées. Mémoires du Muséum National d'Histoire Naturelle, Série B, Botanique 27: 153-252

Pichon M. 1949. Classification des Apocynacées: XXVII, Détermination des graines de Plumérioidées et de Cerbérioidées. Bulletin du Muséum National d'Histoire Naturelle, Série 2. 21: 266-269.

Pichon M. 1950a. Classification des Apocynacées. XXVIII. Supplément aux Pluméeriodées. Mémoires du Muséum National d'Histoire Naturelle, Série Botanique 1: 145-173.

Pichon M. 1950b. Classification des Apocynacées: XXXI. Le fruit des genres Thevetia et Ahovai. Bulletin $d u$ Muséum National d'Histoire Naturelle, Série 2. 22: 291-294.

Pimm SL, Jenkins CN, Abell R, Brooks TM, Gittleman JL, Joppa LN, Sexton JO. 2014. The biodiversity of species and their rates of extinction, distribution, and protection. Science 344: 1246752. DOI: https://doi.org/10.1126/ science. 1246752

Ping-Tao L, Leeuwenberg AJM, Middleton DJ. 1995a. Apocynaceae. Flora of China 16: 143-188.

Ping-Tao L, Gilbert MG, Stevens WD. $1995 \mathrm{~b}$. Asclepiadaceae. Flora of China 16: 189-270.

Portal de Datos Abiertos UNAM. 2019. https:// datosabiertos.unam.mx (accessed February, 2019).

Potgieter K, Albert VA. 2001. Phylogenetic relationships within Apocynaceae s.l. based on trnL intron and trnL-F spacer sequences and propagule characters. Annals of the Missouri Botanical Garden 88: 523-549. DOI: https:// doi.org/10.2307/3298632

Ramos-Silva A, Tavares-Carreón F, Figueroa M, De la Torre-Zavala S, Gastelum-Arellanez A, RodríguezGarcía A, Avilés-Arnaut H. 2017. Anticancer potential of Thevetia peruviana fruit methanolic extract. BMC Complementary and Alternative Medicine 17: 241. DOI: https://doi.org/10.1186/s12906-017-1727-y

Rapini A. 2012. Taxonomy "under construction": advances in the systematics of Apocynaceae, with emphasis on the Brazilian Asclepiadoideae. Rodriguésia 63: 75-88. DOI: http://doi.org/10.1590/S2175-78602012000100007

Rapini A, Chase MW, Konno TU. 2006. Phylogenetics of South American Asclepiadoideae (Apocynaceae). Taxon 55: 119-124. DOI: https://doi.org/10.2307/25065533

Rascón-Valenzuela LA, Jiménez-Estrada M, VelázquezContreras CA, Garibay Escobar A, Medina-Juárez LA, Gámez-Meza N, Robles-Zepeda RE. 2015. Antiproliferative and apoptotic activities of extracts of Asclepias subulata. Pharmaceutical biology 53: 
1741-1751. DOI: https://doi.org/10.3109/13880209.201 5.1005752

Raxworthy CJ, Ingram CM, Rabibisoa N, Pearson RG. 2007. Applications of ecological niche modeling for species delimitation: a review and empirical evaluation using day geckos (Phelsuma) from Madagascar. Systematic Biology 56: 907-923. DOI: https://doi.org/ $\underline{10.1080 / 10635150701775111}$

Rodríguez-Morales LO. 2015. Revisión taxonómica de la subfamilia Asclepiadoideae (Apocynaceae) en el estado de Morelos. Tesis de Lic. Universidad Autónoma del Estado de Morelos.

Rowley GD. 1984. Jacquin and the Stapelieae. Bradleya 1984: 1-9. DOI: https://doi.org/10.25223/brad.n2.198 4.a1

Rzedowski J, Calderón de Rzedowski G. 1998. Apocynaceae. Flora del Bajío y de Regiones Adyacentes. 70: $1-63$.

Rzedowski J, Calderón de Rzedowski G. 2013. Datos para la apreciación de la flora fanerogámica del bosque tropical caducifolio de México. Acta Botanica Mexicana 102: 1-23. DOI: https://doi.org/10.21829/abm102.201 $\underline{3.229}$

Sahagún B. 1577. Historia general de las cosas de Nueva España. El Códice Florentino. Libro XI: de las cosas naturales. México: Imprenta del ciudadano A. Valdés.

SEMARNAT [Secretaría del Medio Ambiente y Recursos Naturales]. 2010a. Norma Oficial Mexicana NOM-059SEMARNAT-2010, Protección ambiental - Especies nativas de México de flora y fauna silvestres - Categorías de riesgo y especificaciones para su inclusión, exclusión o cambio - Lista de especies en riesgo. Diario Oficial de la Federación. 2da Sección, 30 de diciembre de 2010.

SEMARNAT [Secretaría del Medio Ambiente y Recursos Naturales]. 2010b. Modificación. Norma Oficial Mexicana NOM-059-SEMARNAT-2010, - Especies nativas de México de flora y fauna silvestres - Categorías de riesgo y especificaciones para su inclusión, exclusión o cambio - Lista de especies en riesgo. Diario Oficial de la Federación, 3ra Edición. México. (Modificado 14/11/2019).

Sennblad B, Bremer B. 1996. The familial and subfamilial relationships of Apocynaceae and Asclepiadaceae evaluated with $r b c L$ data. Plant Systematics and Evolution, 202: 153-175. DOI: https://doi.org/10.1007/ BF00983380

Sennblad B, Bremer B. 2000. Is there a justification for differential a priori weighting in coding sequences? A case study from rbcL and Apocynaceae sl. Systematic Biology 49: 101-113. DOI: https://doi.org/10.1080/1063 5150050207410

Sennblad B, Endress ME, Bremer B. 1998. Morphology and molecular data in phylogenetic fraternity: the tribe
Wrightieae (Apocynaceae) revisited. American Journal of Botany 85: 1143-1158. DOI: https://doi.org/ $\underline{10.2307 / 2446347}$

Sessé M, Mociño JM. 1893. Plantae Novae Hispaniae. México: Oficina Tip. de la Secretaría de Fomento, San Andrés núm. 15. https://bibdigital.rjb.csic.es/idurl/ $\underline{1 / 12069}$

Sessé M, Mociño JM. 1894. Flora mexicana. México. Oficina Tipográfica de la Secretaría de Fomento. DOI: https://doi.org/10.5962/bhl.title.119200

Shreve F, Wiggins I. 1964. Vegetation and flora of the Sonoran desert. Vol. 2. California. Stanford University Press. ISBN: 978-080-4701-63-1

Simões AO, Endress ME, van der Niet T, Kinoshita LS, Conti E. 2004. Tribal and intergeneric relationships of Mesechiteae (Apocynoideae, Apocynaceae): evidence from three noncoding plastid DNA regions and morphology. American Journal of Botany 91: 1409-1418. DOI: https://doi.org/10.3732/ajb.91.9.1409

Simões AO, Endress ME, van der Niet T, Kinoshita LS, Conti E. 2006. Is Mandevilla (Apocynaceae, Mesechiteae) monophyletic? Evidence from five plastid DNA loci and morphology. Annals of the Missouri Botanical Garden 93: 565-591. DOI: http://dx.doi.org/ 10.3417/0026-6493(2006)93[565:IMAMME]2.0.CO;2

Simões AO, Livshultz T, Conti E, Endress ME. 2007. Phylogeny and systematics of the Rauvolfioideae (Apocynaceae) based on molecular and morphological evidence. Annals of the Missouri Botanical Garden 94: 268-297. DOI: https://doi.org/10.3417/0026-6493(2007) 94[268:PASOTR]2.0.CO;2

Simões AO, Endress ME, Conti E. 2010. Systematics and character evolution of Tabernaemontaneae (Apocynaceae, Rauvolfioideae) based on molecular and morphological evidence. Taxon 59: 772-790. DOI: https://doi.org/10.1002/tax.593009

Simões AO, Kinoshita LS, Koch I, Silva MJ, Endress ME. 2016. Systematics and character evolution of Vinceae (Apocynaceae). Taxon 65: 99-122. DOI: https://doi.org/ $\underline{10.12705 / 651.7}$

Smith CE. 1967. Plants remains. In: Byers DS, ed. The Prehistory of the Tehuacan Valley. Environment and subsistence. Vol. 1. Austin. Robert S. Peabody Foundation, Phillips Academy, Andover University of Texas. ISBN: 978-029-2736-83-2

Smithsonian National Museum of Natural History. 2019. http://www.mnh.si.edu (accessed Jan 2019).

Solano E, Feria TP. 2006. Ecological niche modeling and geographic distribution of the genus Polianthes L. (Agavaceae) in Mexico: using niche modeling to improve assessments of risk status. Plant conservation and biodiversity 6:1885-1900. DOI: https://doi.org/ $\underline{10.1007 / \mathrm{s} 10531-006-9091-0}$ 
Sosa V, Gómez-Pompa A. 1994. Lista florística. Flora de Veracruz. Fascículo 82. México: Instituto de Ecología, AC Xalapa, Ver. University of California, Riverside, CA. ISBN: 968-7213-59.0

Sosa V, De-Nova JA, Vásquez-Cruz M. 2018. Evolutionary history of the flora of Mexico: Dry forests cradles and museums of endemism. Journal of Systematics and Evolution 56: 523-536. DOI: https://doi.org/10.1111/ jse. 12416

Sousa M. 1969. Las colecciones botánicas de C.A. Purpus en México, periodo 1898-1925. University of California Press. ISBN-10: 0520090241 ISBN-13: 978-0520090248

Sousa M. 1979. Itinerario botánico de G. Andrieux en México. Taxon 28: 97-102. DOI: https://doi.org/ $\underline{10.2307 / 1219564}$

Standley PC. 1924. Apocynaceae. In: Standley, P.C. (Ed.). Trees and shrubs of Mexico. Nuevo México, USA: College of Agriculture and Mechanicals Arts. 23: 1147-1166. DOI: $\underline{\text { https://doi.org/10.5962/bhl.title.15726 }}$

Standley PC. 1926. New Plants from Chiapas by C. A. Purpus. Journal of the Washington Academy of Sciences 16: $14-18$.

Stevens PF. 2001. Angiosperm Phylogeny Website. Version 14, July 2017 [and more or less continuously updated since]." will do. http://www.mobot.org/MOBOT/ research/APweb/.

Stevens WD. 1975. Notes on the genus Matelea (Apocynaceae s. 1.). Phytologia 32: 387-406.

Stevens WD. 1988. A synopsis of Matelea subg. Dictyanthus (Apocynaceae: Asclepiadoideae). Annals of the Missouri Botanical Garden 47: 1533-1564. DOI: https://doi.org/10.2307/2399300

Stevens WD. 1999. Two new combinations in Apocynaceae, Asclepiadoideae from Mesoamerica. Novon 9: 564-564. DOI: https://doi.org/10.2307/3392167

Stevens WD. 2000. New and interesting milkweeds (Apocynaceae, Asclepiadoideae). Novon 10: 242-256. DOI: https://doi.org/10.2307/3393108

Stevens WD. 2001a. Asclepiadaceae. In: Calderón de Rzedowski G, Rzedowski J., eds. Flora Fanerogámica del Valle de México. Pátzcuaro. Michoacán: Instituto de Ecología AC. Comisión Nacional para el Conocimiento y Uso de la Biodiversidad, pp: 564-576. ISBN 970-9000-17-9

Stevens WD. 2001b. Asclepiadaceae R. Br. In: Stevens WD, Ulloa UC, Pool A, Montiel OM, eds. Flora de Nicaragua 1. St. Louis: Monographs in Systematic Botany Missouri Botanical Garden, pp. 234-270. ISBN 10: 0915279959; ISBN 13: 9780915279951

Stevens WD. 2005. Novelties in Cynanchum L., sensu Woodson, in Mesoamerica. Novon 15: 620-641.
Stevens WD. 2018. Vailia anomala, a new name for Blepharodon mucronatum (Apocynaceae, Asclepiadoideae). Phytoneuron 3: 1-5.

Stevens WD, Morales JF. 2009. Apocynaceae. In: Davidse G, Sousa SM, Knapp M, Chiang F, Barrie FR, eds. Flora Mesoamericana: Cucurbitaceae a Polemoniaceae. México: Universidad Nacional Autónoma de México Instituto de Biología; Saint Louis: Missouri Botanical Garden; London: The Natural History Museum, pp. 662-768. ISBN 979-607-02-0901-7

Sundell E. 1981. The New World species of Cynanchum subgenus Mellichampia (Asclepiadaceae). Evolutionary Monographs 5: 1-62. DOI: https://doi.org/10.2307/ $\underline{2806043}$

The Milkweed Zoo. 2019. https://www.flickr.com/groups/ 73512350@N00/ (accessed May 2019).

Thiers B. 2019. Index herbariorum: a global directory of public herbaria and associated staff. New York Botanical Garden's Virtual Herbarium. Available at: http://sweetgum.nybg.org/ih (Accessed February, 2019)

Thomas CD, Cameron A, Green RE, Bakkenes M, Beaumont LJ, Collingham YC, Erasmus BFN, Ferreira de Siqueira M, Grainger A, Hannah L, Hughes L, Huntley B, van Jaarsveld AS, Midgley GF, Miles L, Ortega-Huerta MA, Peterson T, Phillips OL, Williams SE. 2004. Extinction risk from climate change. Nature 427: 145. DOI: https://doi.org/10.1038/nature02121

Tropicos 2019. http://www.tropicos.org (accessed Jan 2019)

Villaseñor JL. 2015. ¿La crisis de la biodiversidad es la crisis de la taxonomía? Botanical Sciences 93: 3-14. DOI: https://doi.org/10.17129/botsci.456

Villaseñor JL. 2016. Checklist of the native vascular plants of Mexico. Revista Mexicana de Biodiversidad 87: 559-902. DOI: http://doi.org/10.1016/j.rmb.2016.06.017

Villaseñor, JL. 2018. Diversidad y distribución de la familia Asteraceae en México. Botanical Sciences 96: 332-358. DOI: https://doi.org/10.17129/botsci.1872

Wandersee JH, Schussler EE. 1999. Preventing plant blindness. The American Biology Teacher 61: 84-86. DOI: https://doi.org/10.2307/4450624

Wiggins IL. 1980. Flora of Baja California. Stanford University Press, California. ISBN: 978-080-4710-16-9

Williams JK. 1995. Miscellaneous notes on Haplophyton (Apocynaceae: Plumerieae: Haplophytinae). SIDA, Contributions to Botany 16: 469-475.

Williams JK. 1996. The Mexican genera of the Apocynaceae (sensu A. DC.), with key and additional taxonomic notes. SIDA, Contributions to Botany 17: 197-213.

Williams JK. 1998. A revision of Thenardia HBK (Apocynaceae, Apocynoideae). Lundellia 1: 78-95. DOI: https://doi.org/10.25224/1097-993X-1.1.78 
Williams JK. 1999. A phylogenetic and taxonomic study of the Apocynaceae subfamily Apocynoideae of Mexico with a synopsis of subfamily Plumerioideae. Ph.D. Thesis. The University of Texas.

Williams JK. 2002a. Thoreauea (Apocynaceae: Apocynoideae), a new genus from Oaxaca, Mexico. Lundellia 5: 47-58. DOI: https://doi.org/10.25224/ 1097-993X-5.1.47

Williams JK. 2002b. A further evaluation of Echites sect. Yucatanense (Apocynaceae) with additional notes on the genus. Brittonia 54: 310-317. DOI: https://doi.org/ 10.1663/0007-196X(2003)54[310:AFEOES]2.0.CO;2

Williams JK. 2004. Polyphyly of the genus Echites (Apocynaceae: Apocynoideae: Echiteae): Evidence based on a morphological cladistic analysis. SIDA, Contributions to Botany 21: 117-131.

Wong SK, Lim YY, Chan EW. 2013. Botany, uses, phytochemistry and pharmacology of selected Apocynaceae species: A review. Pharmacognosy Communications 3: 2-11. DOI: https://doi.org/10.5530/ pc.2013.3.2

Woodson RE. 1928a. Studies in the Apocynaceae, II, A revision of the genus Stemmadenia. Annals of the Missouri Botanical Garden 15: 341-379. DOI: https:// doi.org/10.2307/2394021

Woodson RE. 1928b. Studies in the Apocynaceae. III. A Monograph of the genus Amsonia. Annals of the Missouri Botanical Garden 15: 379-434. DOI: https:// doi.org/10.2307/2394022

Woodson RE. 1932a. The identity and nomenclature of Apocynum androsaemifolium L. Rhodora 34: 30-31. https://www.biodiversitylibrary.org/page/602984

Woodson RE. 1932b. New or otherwise noteworthy Apocynaceae of tropical America. II. Annals of the Missouri Botanical Garden 19: 45-76. DOI: https:// doi.org/10.2307/2394172

Woodson RE. 1933. Studies in the Apocynaceae. IV. The American genera of Echitoideae. Annals of the Missouri

\section{Editor de sección: Monserrat Vázquez Sánchez}

Contribución de los autores: Todos los autores contribuyeron en la curación del listado de Apocynaceae de México, así como de las colecciones revisadas, la redacción y revisión del manuscrito. LOAC coordinación, diseño, redacción, compilación y revisión. LLP síntesis de datos y redacción. CSIH, EBC, KGMM, MGCH redacción, compilación de la bibliografía relevante y correcciones.
Botanical Garden 20: 605-790. DOI: https://doi.org/ $\underline{10.2307 / 2394198}$

Woodson RE. 1934. New or otherwise noteworthy Apocynaceae of tropical America. IV. Annals of the Missouri Botanical Garden 21: 613-623. DOI: https:// doi.org/10.2307/2394188

Woodson RE. 1935. Studies in the Apocynaceae. IV. The American Genera of Echitoideae. Annals of the Missouri Botanical Garden 22: 153-306. DOI: https://doi.org/ $\underline{10.2307 / 2394156}$

Woodson RE. 1938. North American Flora, Apocynaceae. New York Botanical Garden Press 29: 103-192.

Woodson RE. 1939. New or otherwise noteworthy Apocynaceae of Tropical America. VII. Annals of the Missouri Botanical Garden 26: 257-259. DOI: https:// doi.org/10.2307/2394294

Woodson, RE. 1940. The apocynaceous flora of the Yucatan Peninsula. Publications of the Carnegie Institution of Washington 522: 59-162.

Woodson RE. 1941. The North American Asclepiadaceae. I. Perspective of the genera. Annals of the Missouri Botanical Garden. 28: 193-244. DOI: https://doi.org/ $\underline{10.2307 / 2394270}$

Woodson RE. 1945. Notes on some North American asclepiads. Annals of the Missouri Botanical Garden 32: 369-371. DOI: https://doi.org/10.2307/2394381

Yang LL, Li HL, Wei L, Yang T, Kuang DY, Li MH, Liao YY, Chen ZD, Wu H, Zhang SZ. 2016. A supermatrix approach provides a comprehensive genus-level phylogeny for Gentianales. Journal of Systematics and Evolution 54: 400-415. DOI: https://doi.org/10.1111/ jse. 12192

Zuccarini JG. 1846. Plantarum novarum vel minus cognitarum, quae in horto botánico herbarioque regio Monacensi servantur. Abhandlungen der MathematischPhysikalischen Classe der Königlich Bayerischen Akademie der Wissenschaften 4: 1-12. https://www. biodiversitylibrary.org/page/12548912 